\title{
Application of Chebyshev tau method for bending analysis of elastically restrained edge functionally graded nano/micro-scaled sandwich beams, under non-uniform normal and shear loads
}

\author{
M. Matinfar ${ }^{1} \cdot$ M. Mahdavi Shirazi ${ }^{1} \cdot$ M. M. Alipour ${ }^{2}$
}

Received: 13 May 2019 / Accepted: 25 September 2019 / Published online: 5 October 2019

(c) The Author(s) 2019

\begin{abstract}
In this study, for the first time, an approximate solution procedure based on the Chebyshev tau method (CTM) is developed for bending analysis of functionally graded nano/micro-scaled sandwich beams. The proposed approach has the advantage of decreasing the problem to the solution of a system of algebraic equations, which may then be solved by any numerical method. In the CTM, the solution is approximated via a truncated Chebyshev series expansion and the Chebyshev polynomials are used as the test function. Based on the proposed technique, sandwich beams with elastically restrained edges under arbitrary non-uniform distributed normal and shear loads can be analyzed. The effectiveness of the CTM is illustrated by comparison of the obtained results for various end supports with those extracted from the ABAQUS software. In each considered cases, the numerical results indicate that the proposed scheme is of high accuracy and is efficient for solving the ordinary differential equations and systems of them.
\end{abstract}

Keywords Chebyshev tau method $\cdot$ Chebyshev polynomials $\cdot$ Spectral method $\cdot$ Micro/nano-sandwich beam $\cdot$ Non-uniform loads

\section{Introduction}

Nowadays, spectral methods are extensively utilized for various applications [1]. Their main appeal relies on their premiere rate of convergence for sufficiently smooth functions [2]. Chebyshev tau method is a particularly efficient spectral scheme in which Chebyshev polynomials are used in the tau method of Lanczos [3]. Numerical programs using this technique are often considerably faster with greater accuracy than other standard methods such as finite differencing [4]. Recently, there have been several published papers on the

M. Mahdavi Shirazi

m.mahdavi@stu.umz.ac.ir

M. Matinfar

m.matinfar@umz.ac.ir

M. M. Alipour

m.mollaalipour@umz.ac.ir

1 Department of Mathematics, Faculty of Mathematical Sciences, University of Mazandaran, Babolsar, Iran

2 Department of Mechanical Engineering, University of Mazandaran, Babolsar, Iran applications of the tau method. Siyyam and Syam [5] presented the CTM for the two-dimensional Poisson equation. Ahmadi and Adibi [6] applied the Chebyshev tau method for the Laplace equation. Saadatmandi and Dehghan [7] utilized the CTM to approximate the solution of hyperbolic telegraph problem. Wang [8] applied a time-splitting CT spectral method to the Ginzburg-Landau-Schrödinger equation with zero/nonzero far-field boundary conditions. Lee [9] applied a Chebyshev tau method based on Euler-Bernoulli and Timoshenko beam theories to the free vibration analyses of stepped beams. Functionally graded materials (FGMs) have great practical applications in engineering and industrial fields [10] and represent a novel generation of materials, composed of a mixture of two different materials (metal and ceramic) [11]. They are constructed by a continuous change in composition and do not possess a particular interface [12]. FGMs concepts are applied to metals, ceramics and organic composites to generate improved components with superior physical properties [27]. Investigations in this emerging area [13-26] are only at the beginning stage and are very confined to most of the previous studies devoted to the compression behavior and energy dissipation performance only [28]. In this study, we employ the Chebyshev 
tau spectral scheme for solution of governing equations of a three-layer sandwich beam regarded to non-uniform normal and shear loads. The paper is organized as follows. In "The tau method" section, the Chebyshev tau spectral method is described briefly. The third section is devoted to solving our intended problem by the CTM and obtaining the approximate results. In "Results and comparisons" section, a detailed discussion is carried out to show the accuracy, validity and applicability of the technique. Finally, we give our conclusions in "Conclusion" section.

\section{The tau method}

Some fundamental results for Chebyshev approximation [29] are needed. The Chebyshev polynomial of degree $n$ on $[-1,1]$ is defined by $T_{n}(\cos \theta)=\cos (n \theta)$. For $x \in[-1,1]$, $\left\{T_{n}(x)\right\}$ satisfy the following recurrence relation

$$
\begin{gathered}
T_{n+1}(x)=2 x T_{n}(x)-T_{n-1}(x), \\
n>0, T_{0}(x)=1, T_{1}(x)=x .
\end{gathered}
$$

They form an orthonormal basis with respect to

$$
(u, v)_{\omega}=\left(\int_{-1}^{1} u(x) v(x) \omega(x) \mathrm{d} x\right),
$$

where the weight function $\omega$ is defined by $\omega(x)=\frac{1}{\sqrt{1-x^{2}}}$.

Proposition 1 The polynomials $T_{n}(x)$ are orthogonal, i.e.,

$\left(T_{n}, T_{m}\right)_{0, \omega}=\frac{\pi}{2} c_{n} \delta_{n, m}, \quad m, n \in \mathbb{N}$,

where $\delta_{n, m}$ is

$\delta_{n, m}= \begin{cases}0, & n \neq m, \\ 1, & n=m,\end{cases}$

and the coefficients $c_{n}$ are

$c_{n}= \begin{cases}2, & n=0 \\ 1, & n>0 .\end{cases}$

Some useful properties of Chebyshev polynomials are [30]

$\left|T_{n}(x)\right| \leq 1, \quad|x| \leq 1, \quad T_{n}( \pm 1)=( \pm 1)^{n}$,

$T_{n}^{\prime}( \pm 1)=( \pm 1)^{n} n^{2}$,

$T_{n}(x) T_{m}(x)=\frac{T_{n+m}(x)+T_{|n-m|}(x)}{2}$.
The Chebyshev expansion of a function $u(x)$ is $u(x)=\sum_{n=0}^{\infty} b_{n} \cdot T_{n}(x)$ where $b_{n}=\frac{\left(u, T_{n}\right)_{0, \infty}}{\left\|T_{n}\right\|_{0, \omega}^{2}}=\frac{2}{\pi c_{n}}\left(u, T_{n}\right)_{0, \omega}$.

Consider the expansion of a function $u(x)$ or its derivatives in terms of Chebyshev polynomials on the interval $[-1,1]$. Suppose that $u$ and its derivatives can be expanded as

$$
\begin{aligned}
& u(x)=\sum_{n=0}^{\infty} b_{n}^{(0)} T_{n}(x), \\
& \frac{d^{m} u}{\mathrm{~d} x^{m}}=\sum_{n=0}^{\infty} b_{n}^{(m)} T_{n}(x), m=0,1, \ldots .
\end{aligned}
$$

Then, recursion relation $c_{n-1} b_{n-1}^{(m)}=2 n b_{n}^{(m-1)}+b_{n+1}^{(m)}, n \geq 1$ gives

$$
\begin{aligned}
& c_{n} b_{n}^{(1)}=2 \sum_{\substack{p=n+1 \\
p+n \text { odd }}}^{\infty} p b_{p}, \quad n \geq 0, \\
& c_{n} b_{n}^{(2)}=2 \sum_{\substack{p=n+2 \\
p+n \text { even }}}^{\infty} p\left[p^{2}-n^{2}\right] b_{p}, \quad n \geq 0,
\end{aligned}
$$

where $c_{n}$ is defined by (5). The formulae given above are utilized to expand products of Chebyshev polynomials and derivatives of Chebyshev polynomials as expansions in Chebyshev polynomials. For instance, if a function $u(x)$ and its first and second derivatives $u^{\prime}(x)$ and $u^{\prime \prime}(x)$ have series expansions in terms of Chebyshev polynomials

$$
\begin{aligned}
& u(x)=\sum_{n=0}^{N} b_{n} T_{n}(x), u^{\prime}(x)=\sum_{n=0}^{N-1} b_{n}^{(1)} T_{n}(x), \\
& u^{\prime \prime}(x)=\sum_{n=0}^{N-2} b_{n}^{(2)} T_{n}(x),
\end{aligned}
$$

then the coefficients $b_{n}^{(1)}$ and $b_{n}^{(2)}$ are related to the coefficients $b_{n}$, by

$$
c_{n} b_{n}^{(1)}=2 \sum_{\substack{p=n+1 \\ p+n \text { odd }}}^{p=N} p b_{p}
$$

$$
c_{n} b_{n}^{(2)}=2 \sum_{\substack{p=n+2 \\ p+n \text { even }}}^{p=N} p\left[p^{2}-n^{2}\right] b_{p}, \quad n \geq 0,
$$


where $c_{n}$ is defined by (5).

The tau approach was first suggested by Lanczos [3, 31] , and its use with Chebyshev polynomials was later developed widely by Fox [32] and was applied by Orszag for an extensive variety of problems [33-35]. Consider the following linear two-point boundary value problem

(LP) $\left\{\begin{array}{l}L u=f, x \in(-1,1), \\ B u=0,\end{array}\right.$

where $L$ is a linear differential operator acting in a Hilbert space $X$ and $B$ stands for a set of linear differential operators defined on -1 and 1 . The Chebyshev tau method is characterized by the following choice:

$X=L_{\omega}^{2}(-1,1), X_{N}=\left\{v \in \mathcal{P}_{N} \mid B v=0\right\}, Y_{N}=\mathcal{P}_{N-\beta}$,

where $X_{N}$ denotes the space of trial functions and $Y_{N}$ is that of test functions. Moreover, $\mathcal{P}_{N}$ is the space of algebraic polynomials of degree at most $N \in \mathbb{N}, N>0$ and $\beta$ stands for the number of boundary conditions. Accepting the family $\left\{T_{n}, n=0,1,2, \ldots, N\right\}$ as a basis for the finite dimensional space $X_{N}$ and the family $\left\{T_{m}, m=0,1,2, \ldots, N-\beta\right\}$ as a set of test functions in $Y_{N}$, the variational formulation corresponding to $(L P)$ is:

(CT) $\left\{\begin{array}{l}\text { find the coefficients } b_{n} \text { of } u_{N}(x) \in \mathcal{P}_{N}, \\ u_{N}(x)=\sum_{n=0}^{N} b_{n} \cdot T_{n}(x), \text { such that } \\ \int_{-1}^{1}\left(L u_{N}(x)-f(x)\right) \psi_{m} \mathrm{~d} x=0, \quad m=0,1,2, \ldots, N-\beta, \\ \text { and } \sum_{n=0}^{N} b_{n} \cdot B\left(T_{n}\right)=0,\end{array}\right.$ where

$\psi_{m}(x)=\frac{2}{\pi c_{m}} T_{m}(x) \omega(x)=\frac{2}{\pi c_{m}} T_{m}(x) \frac{1}{\sqrt{1-x^{2}}}$.

\section{Governing equations of the FG micro/ nano-sandwich beams}

A three-layer sandwich beam with regard to non-uniform normal and shear loads is investigated, as shown in Fig. 1. The thickness of the top and bottom face sheets and core are denoted, respectively, by $h_{t}, h_{b}$ and $h_{c}$. The general boundary conditions are simulated by employing the in-plane and transverse translational springs at the mid-plane of the core and the rotational springs at the mid-plane of each layer. By setting the spring coefficients equal to infinite or zero, the classical boundary conditions can be modeled as some particular cases of the elastic supports. Based on the theory of layerwise [36-38], the displacement field of the layers can be expressed as:

$\begin{cases}u_{t}=u_{0}+\left(z-\frac{h_{c}}{2}\right) \varphi_{x}^{(t)}+\frac{h_{c}}{2} \varphi_{x}^{(c)}, & \frac{h_{c}}{2} \leq z \leq \frac{h_{c}}{2}+h_{t}, \\ u_{c}=u_{0}+z \varphi_{x}^{(c)}, & -\frac{h_{c}}{2} \leq z \leq \frac{h_{c}}{2}, \\ u_{b}=u_{0}+\left(z+\frac{h_{c}}{2}\right) \varphi_{x}^{(b)}-\frac{h_{c}}{2} \varphi_{x}^{(c)}, & -h_{b}-\frac{h_{c}}{2} \leq z \leq-\frac{h_{c}}{2}, \\ w=w_{0}, & \end{cases}$

where $u_{t}, u_{c}$ and $u_{b}$ are the in-plane displacement of the top, core and bottom layers, $\varphi_{x}^{(t)}, \varphi_{x}^{(c)}$ and $\varphi_{x}^{(b)}$ are the rotation of the section of the top, core and bottom layers, and $w$ is the
Fig. 1 Schematic diagram of an elastically restrained sandwich beam under arbitrary non-uniform normal and shear loads

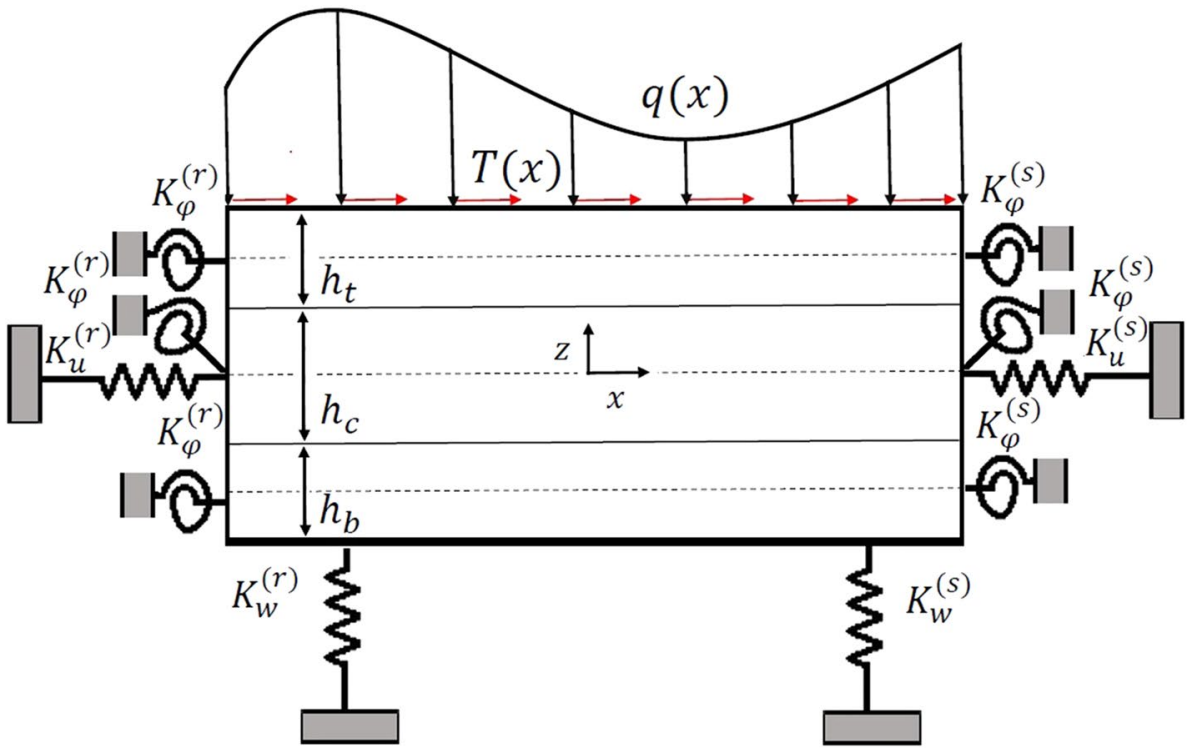


transverse displacement of sandwich beam. For small deflections of beam, strains of each layer are given as:

$\varepsilon_{x}^{(i)}=\frac{\partial u_{i}}{\partial x}, \quad \gamma_{x z}^{(i)}=\frac{\partial u_{i}}{\partial z}+\frac{\partial w_{i}}{\partial x}, \quad i=t, c, b$.

Constitutive model that expresses the nonlocal stress tensor is as follow:

$$
\begin{aligned}
& \left(1-\mu \nabla^{2}\right) \sigma_{x}^{(i)}=\frac{E_{i}}{1-v_{i}^{2}} \varepsilon_{x}^{(i)}, \\
& \left(1-\mu \nabla^{2}\right) \tau_{x z}^{(i)}=\frac{E_{i}}{2\left(1+v_{i}\right)} \gamma_{x z}^{(i)} \quad i=t, c, b .
\end{aligned}
$$

Based on the minimum total potential energy principle, the governing equations of the elastically restrained sandwich beams may be derived as:

$\delta \Pi=\delta U-\delta W=0$,

where $W$ is the work of the externally applied loads and $U$ is the potential energy.

$$
\begin{aligned}
\delta U= & \int_{A} \int_{\frac{h_{c}}{2}}^{\frac{h_{c}}{2}+h_{t}}\left(\sigma_{x}^{(t)} \delta \varepsilon_{x}^{(t)}+\tau_{x z}^{(t)} \delta \gamma_{x z}^{(t)}\right) \mathrm{d} z \mathrm{~d} A \\
& +\int_{A} \int_{-\frac{h_{c}}{2}}^{\frac{h_{c}}{2}}\left(\sigma_{x}^{(c)} \delta \varepsilon_{x}^{(c)}+\tau_{x z}^{(c)} \delta \gamma_{x z}^{(c)}\right) \mathrm{d} z \mathrm{~d} A \\
& +\int_{A} \int_{-\frac{h_{c}}{2}-h_{b}}^{-\frac{h_{c}}{2}}\left(\sigma_{x}^{(b)} \delta \varepsilon_{x}^{(b)}+\tau_{x z}^{(b)} \delta \gamma_{x z}^{(b)}\right) \mathrm{d} z \mathrm{~d} A,
\end{aligned}
$$

$$
\begin{aligned}
\delta W= & \int_{A}\left(\left.T \delta u_{t}\right|_{z=\frac{h_{c}}{2}+h_{t}}+q \delta w\right) d A+\left.V_{t} \delta w\right|_{x=r}+\left.V_{c} \delta w\right|_{x=s} \\
& +\left.K_{u}^{(r)} \delta u_{0}\right|_{x=r}+\left.K_{u}^{(s)} \delta u_{0}\right|_{x=s}+\left.K_{w}^{(r)} \delta w\right|_{x=r}+\left.K_{w}^{(s)} \delta w\right|_{x=s} \\
& +\left.K_{\varphi}^{(r)}\left(\delta \varphi^{(t)}+\delta \varphi^{(c)}+\delta \varphi^{(b)}\right)\right|_{x=r} \\
& +\left.K_{\varphi}^{(s)}\left(\delta \varphi^{(t)}+\delta \varphi^{(c)}+\delta \varphi^{(b)}\right)\right|_{x=s}
\end{aligned}
$$

where $K_{u}^{(i)}, K_{\varphi}^{(i)}$ and $K_{w}^{(i)}, i=r, s$, are the stiffness parameters of the in-plane translational, rotational and transverse translational springs. $q(x)$ and $T(x)$ are the arbitrary distributed transverse and shear loads of the top surface of the sandwich beam, respectively. Substituting Eqs. (20) and (21) into Eq. (19) and using Eqs. (17) and (18), by some manipulation the governing differential equations of the FG sandwich micro/ nano-beam may be expressed as:

$$
\begin{aligned}
& \left(A^{(t)}+A^{(c)}+A^{(b)}\right) u_{0, x x}+\left(B^{(t)}-\frac{h_{2}}{2} A^{(t)}\right) \varphi_{x, x x}^{(t)} \\
& +\left(\frac{h_{c}}{2} A^{(t)}+B^{(c)}-\frac{h_{c}}{2} A^{(b)}\right) \varphi_{x, x x}^{(c)} \\
& +\left(B^{(b)}+\frac{h_{c}}{2} A^{(b)}\right) \varphi_{x, x x}^{(b)}=-\left(1-\mu \nabla^{2}\right) T, \\
& \left(B^{(t)}-\frac{h_{c}}{2} A^{(t)}\right) u_{0, x x}+\left(D^{(t)}-h_{c} B^{(t)}+\frac{h_{c}^{2}}{4} A^{(t)}\right) \varphi_{x, x x}^{(t)} \\
& +\left(\frac{h_{c}}{2} B^{(t)}-\frac{h_{c}^{2}}{4} A^{(t)}\right) \varphi_{x, x x}^{(c)}-\bar{A}^{(t)}\left(\varphi_{x}^{(t)}+w_{, x}\right) \\
& =-h_{t}\left(1-\mu \nabla^{2}\right) T \text {, } \\
& \left(\frac{h_{c}}{2} A^{(t)}+B^{(c)}-\frac{h_{c}}{2} A^{(b)}\right) u_{0, x x}+\frac{h_{c}}{2}\left(B^{(t)}-\frac{h_{c}}{2} A^{(t)}\right) \varphi_{x, x x}^{(t)} \\
& +\left(\frac{h_{c}^{2}}{4} A^{(t)}+D^{(c)}+\frac{h_{c}^{2}}{4} A^{(b)}\right) \varphi_{x, x x}^{(c)}-\frac{h_{c}}{2}\left(B^{(b)}+\frac{h_{c}}{2} A^{(b)}\right) \varphi_{x, x x}^{(b)} \\
& -\bar{A}^{(c)}\left(\varphi_{x}^{(c)}+w_{, x}\right)=-\frac{h_{c}}{2}\left(1-\mu \nabla^{2}\right) T, \\
& \left(B^{(b)}+\frac{h_{c}}{2} A^{(b)}\right) u_{0, x x}-\frac{h_{c}}{2}\left(B^{(b)}+\frac{h_{c}}{2} A^{(b)}\right) \varphi_{x, x x}^{(c)} \\
& +\left(\frac{h_{c}^{2}}{4} A^{(b)}+h_{c} B^{(b)}+D^{(b)}\right) \varphi_{x, x x}^{(b)}-\bar{A}^{(b)}\left(\varphi_{x}^{(b)}+w_{, x}\right)=0, \\
& \bar{A}^{(t)} \varphi_{x, x}^{(t)}+\bar{A}^{(c)} \varphi_{x, x}^{(c)}+\bar{A}^{(b)} \varphi_{x, x}^{(b)}+\left(\bar{A}^{(t)}+\bar{A}^{(c)}+\bar{A}^{(b)}\right) \\
& w_{, x x}=\left(1-\mu \nabla^{2}\right) q,
\end{aligned}
$$

where $x \in[r, s]=[-1,1]$ and $\mu=\left(e_{0} \cdot a\right)^{2}$ are the nonlocal parameter which incorporates the small-scale effect, $a$ is the internal characteristic length and $e_{0}$ is a material constant. $A^{(i)}, B^{(i)}, D^{(i)}$ and $\bar{A}^{(i)}$ for $i=t, c, b$ are defined as:

$$
\begin{aligned}
& \left\{\begin{array}{c}
A^{(t)} \\
B^{(t)} \\
D^{(t)}
\end{array}\right\}=\int_{\frac{h_{c}}{2}}^{\frac{h_{c}}{2}+h_{t}} \frac{E_{t}}{1-v_{t}^{2}}\left\{\begin{array}{c}
1 \\
z \\
z^{2}
\end{array}\right\} \mathrm{d} z, \\
& \left\{\begin{array}{c}
A^{(c)} \\
B^{(c)} \\
D^{(c)}
\end{array}\right\}=\int_{-\frac{h_{c}}{2}}^{\frac{h_{c}}{1-v_{c}^{2}}}\left\{\begin{array}{c}
1 \\
z \\
z^{2}
\end{array}\right\} \mathrm{d} z,
\end{aligned}
$$


$\bar{A}^{(c)}=\int_{-\frac{h_{c}}{2}}^{\frac{h_{c}}{2}} \frac{E_{c}}{2\left(1+v_{c}\right)} \mathrm{d} z$,

$\bar{A}^{(b)}=\int_{-\frac{h_{c}}{2}-h_{b}}^{-\frac{h_{c}}{2}} \frac{E_{b}}{2\left(1+v_{b}\right)} \mathrm{d} z$,

where $E_{i}$ and $v_{i}, i=t, c, b$ denote Young's modulus and Poissons ratio of each layer, respectively.

The general boundary conditions can be written as:

$$
\left\{\begin{array}{l}
N_{x}^{(t)}+N_{h_{c}^{(c)}}^{(c)} N_{x}^{(b)}+K_{u}^{(i)} u_{0}=0, \\
M_{x}^{(t)}-\frac{h_{c}}{2} N_{x}^{(t)}+K_{\varphi}^{(i)} \varphi_{x}^{(t)}=0, \\
\frac{h_{c}}{2} N_{x}^{(t)}+M_{x}^{(c)}-\frac{h_{c}}{2} N_{x}^{(b)}+K_{\varphi}^{(i)} \varphi_{x}^{(c)}=0, \\
M_{x}^{(b)}+\frac{h_{c}}{2} N_{x}^{(b)}+K_{\varphi}^{(i)} \varphi_{x}^{(b)}=0, \\
Q_{x}^{(t)}+Q_{x}^{(c)}+Q_{x}^{(b)}+K_{w}^{(i)} w=V, \quad i=r, s,
\end{array}\right.
$$

where $V$ is shear load at the free edge of beam and

$\left\{\begin{array}{l}N_{x}^{(t)}=A^{(t)} u_{0, x}+\left(B^{(t)}-\frac{h_{c}}{2} A^{(t)}\right) \varphi_{x, x}^{(t)}+\frac{h_{c}}{2} A^{(t)} \varphi_{x, x}^{(c)}, \\ M_{x}^{(t)}=B^{(t)} u_{0, x}+\left(D^{(t)}-\frac{h_{c}}{2} B^{(t)}\right) \varphi_{x, x}^{(t)}+\frac{h_{c}}{2} B^{(t)} \varphi_{x, x}^{(c)}\end{array}\right.$

$\left\{\begin{array}{l}N_{x}^{(c)}=A^{(c)} u_{0, x}+B^{(c)} \varphi_{x, x}^{(c)} \\ M_{x}^{(c)}=B^{(c)} u_{0, x}+D^{(c)} \varphi_{x, x}^{(c)}\end{array}\right.$

$\left\{\begin{array}{l}N_{x}^{(b)}=A^{(b)} u_{0, x}+\left(B^{(b)}+\frac{h_{c}}{2} A^{(b)}\right) \varphi_{x, x}^{(b)}-\frac{h_{c}}{2} A^{(b)} \varphi_{x, x}^{(c)} \\ M_{x}^{(b)}=B^{(b)} u_{0, x}+\left(D^{(b)}+\frac{h_{c}}{2} B^{(b)}\right) \varphi_{x, x}^{(b)}-\frac{h_{c}}{2} B^{(b)} \varphi_{x, x}^{(c)}\end{array}\right.$

$\left\{\begin{array}{l}Q_{x}^{(t)}=\bar{A}^{(t)}\left(\varphi_{x}^{(t)}+w_{, x}\right), \\ Q_{x}^{(c)}=\bar{A}^{(c)}\left(\varphi_{x}^{(c)}+w_{, x}\right), \\ Q_{x}^{(b)}=\bar{A}^{(b)}\left(\varphi_{x}^{(b)}+w_{, x}\right) .\end{array}\right.$

Substituting

$\left\{K_{u}^{(r)}, K_{\varphi}^{(r)}, K_{w}^{(r)}, K_{u}^{(s)}, K_{\varphi}^{(s)}, K_{w}^{(s)}\right\}=\{\infty, \infty, \infty, \infty, \infty, \infty\}$,

$\left\{K_{u}^{(r)}, K_{\varphi}^{(r)}, K_{w}^{(r)}, K_{u}^{(s)}, K_{\varphi}^{(s)}, K_{w}^{(s)}\right\}=\{\infty, \infty, \infty, 0,0,0\}$,

$\left\{K_{u}^{(r)}, K_{\varphi}^{(r)}, K_{w}^{(r)}, K_{u}^{(s)}, K_{\varphi}^{(s)}, K_{w}^{(s)}\right\}=\{\infty, \infty, \infty, 0,0, \infty\}$,

in (29), we obtain various edge conditions, respectively, as clamped-clamped edge $(C-C)$ : $\left\{u_{0}( \pm 1)=\varphi_{x}^{(t)}( \pm 1)=\varphi_{x}^{(c)}( \pm 1)=\varphi_{x}^{(b)}( \pm 1)=w( \pm 1)=0\right.$.

clamped-free edge $(C-F)$ :

$\left\{\begin{array}{l}u_{0}(-1)=\varphi_{x}^{(t)}(-1)=\varphi_{x}^{(c)}(-1)=\varphi_{x}^{(b)}(-1)=w(-1)=0, \\ N_{x}^{(t)}(1)+N_{x}^{(c)}(1)+N_{x}^{(b)}(1)=0, \\ M_{x}^{(t)}(1)-\frac{h_{c}}{2} N_{x}^{(t)}(1)=0, \\ \frac{h_{c}}{2} N_{x}^{(t)}(1)+M_{x}^{(c)}(1)-\frac{h_{c}}{2} N_{x}^{(b)}(1)=0, \\ M_{x}^{(b)}(1)+\frac{h_{c}}{2} N_{x}^{(b)}(1)=0, \\ Q_{x}^{(t)}(1)+Q_{x}^{(c)}(1)+Q_{x}^{(b)}(1)=V .\end{array}\right.$

clamped-simply supported edge $(C-S)$ :

$$
\left\{\begin{array}{l}
u_{0}(-1)=\varphi_{x}^{(t)}(-1)=\varphi_{x}^{(c)}(-1)=\varphi_{x}^{(b)}(-1)=w(-1)=0 \\
N_{x}^{(t)}(1)+N_{x}^{(c)}(1)+N_{x}^{(b)}(1)=0, \\
M_{x}^{(t)}(1)-\frac{h_{c}}{2} N_{x}^{(t)}(1)=0 \\
\frac{h_{c}}{2} N_{x}^{(t)}(1)+M_{x}^{(c)}(1)-\frac{h_{c}}{2} N_{x}^{(b)}(1)=0, \\
M_{x}^{(b)}(1)+\frac{h_{c}}{2} N_{x}^{(b)}(1)=0 \\
w(1)=0 .
\end{array}\right.
$$

In order to solve the differential equation system (22) by the CTM, we expand the solution functions $u_{0}, w$ and $\varphi_{x}^{(i)},(i=t, c, b)$ as a finite series of basis functions $\left\{T_{n}(x)\right\}_{n=0}^{N}$ as given below

$\left(u_{0}\right)_{N}(x)=\sum_{n=0}^{N} b_{n} T_{n}(x), w_{N}(x)=\sum_{n=0}^{N} a_{n} T_{n}(x)$,

$\left(\varphi_{x}^{(i)}\right)_{N}(x)=\sum_{n=0}^{N} c j_{n} T_{n}(x), \quad i=t, c, b, \quad j=1,2,3$.

We will need the first- and second-order derivatives of $\left(u_{0}\right)_{N}$, $\left(\varphi_{x}^{(t)}\right)_{N},\left(\varphi_{x}^{(c)}\right)_{N},\left(\varphi_{x}^{(b)}\right)_{N}$ and $w_{N}$ as they relate to $T_{n}(x)$. For this, Eq. (11) will be used.

$$
\begin{aligned}
& \frac{d\left(u_{0}\right)_{N}}{\mathrm{~d} x}=\sum_{n=0}^{N-1} b_{n}^{(1)} T_{n}(x), \frac{d^{2}\left(u_{0}\right)_{N}}{\mathrm{~d} x^{2}}=\sum_{n=0}^{N-2} b_{n}^{(2)} T_{n}(x), \\
& \frac{d\left(\varphi_{x}^{(t)}\right)_{N}}{\mathrm{~d} x}=\sum_{n=0}^{N-1} c 1_{n}^{(1)} T_{n}(x), \frac{d^{2}\left(\varphi_{x}^{(t)}\right)_{N}}{\mathrm{~d} x^{2}}=\sum_{n=0}^{N-2} c 1_{n}^{(2)} T_{n}(x), \\
& \frac{d\left(\varphi_{x}^{(c)}\right)_{N}}{\mathrm{~d} x}=\sum_{n=0}^{N-1} c 2_{n}^{(1)} T_{n}(x), \frac{d^{2}\left(\varphi_{x}^{(c)}\right)_{N}}{\mathrm{~d} x^{2}}=\sum_{n=0}^{N-2} c 2_{n}^{(2)} T_{n}(x), \\
& \frac{d\left(\varphi_{x}^{(b)}\right)_{N}}{\mathrm{~d} x}=\sum_{n=0}^{N-1} c 3_{n}^{(1)} T_{n}(x), \frac{d^{2}\left(\varphi_{x}^{(b)}\right)_{N}}{\mathrm{~d} x^{2}}=\sum_{n=0}^{N-2} c 3_{n}^{(2)} T_{n}(x),
\end{aligned}
$$

$\frac{d w_{N}}{\mathrm{~d} x}=\sum_{n=0}^{N-1} a_{n}^{(1)} T_{n}(x), \frac{d^{2} w_{N}}{\mathrm{~d} x^{2}}=\sum_{n=0}^{N-2} a_{n}^{(2)} T_{n}(x)$. 
Let

$T(x)=t_{0}+t_{1} x+t_{2} x^{2}+t_{3} x^{3}, t_{i} \in \mathbb{R}, i=0,1,2,3$,

$q(x)=q_{0}+q_{1} x+q_{2} x^{2}+q_{3} x^{3}, q_{j} \in \mathbb{R}, j=0,1,2,3$.

Then, we have

$T(x)=t_{0}+\frac{1}{2} t_{2} T_{0}+\left(\frac{3}{4} t_{3}+t_{1}\right) T_{1}+\frac{1}{2} t_{2} T_{2}+\frac{1}{4} t_{3} T_{3}$,
$q(x)=q_{0}+\frac{1}{2} q_{2} T_{0}+\left(\frac{3}{4} q_{3}+q_{1}\right) T_{1}+\frac{1}{2} q_{2} T_{2}+\frac{1}{4} q_{3} T_{3}$. inner product of the obtained system of equations with $\psi_{m}(x)$ where

$$
\begin{aligned}
& \left(T_{n}(x), \psi_{m}(x)\right)=\frac{2}{\pi c_{m}} \int_{-1}^{1} T_{n}(x) T_{m}(x) \frac{1}{\sqrt{1-x^{2}}} \mathrm{~d} x=\delta_{n m}, \\
& m=0,1, \ldots, N-2
\end{aligned}
$$

Let $N=3$. Using the recurrence relationships for the first and second derivative expansion coefficients from Eq. (11) and orthogonality properties of the Chebyshev polynomials, the resulted algebraic equation system leads to

$$
\begin{aligned}
& 4\left(A^{(t)}+A^{(c)}+A^{(b)}\right) b_{2}+4\left(B^{(t)}-\frac{h_{c}}{2} A^{(t)}\right) c 1_{2}+4\left(\frac{h_{c}}{2} A^{(t)}+B^{(c)}-\frac{h_{c}}{2} A^{(b)}\right) c 2_{2} \\
& +4\left(B^{(b)}+\frac{h_{c}}{2} A^{(b)}\right) c 3_{2}+t_{0}+\frac{t_{2}}{2}-2 t_{2} \mu=0,\left(A^{(t)}+A^{(c)}+A^{(b)}\right) b_{3} \\
& +24\left(B^{(t)}-\frac{h_{c}}{2} A^{(t)}\right) c 1_{3}+24\left(\frac{h_{c}}{2} A^{(t)}+B^{(c)}-\frac{h_{c}}{2} A^{(b)}\right) c 2_{3} \\
& +24\left(B^{(b)}+\frac{h_{c}}{2} A^{(b)}\right) c 3_{3}+t_{1}+\frac{3}{4} t_{3}-6 t_{3} \mu=0,4\left(B^{(t)}-\frac{h_{c}}{2} A^{(t)}\right) b_{2} \\
& +4\left(D^{(t)}-h_{c} B^{(t)}+\frac{h_{c}^{2}}{4} A^{(t)}\right) c 1_{2}+4\left(\frac{h_{c}}{2} B^{(t)}-\frac{h_{c}^{2}}{4} A^{(t)}\right) c 2_{2} \\
& -\bar{A}^{(t)}\left(c 1_{0}+a_{1}+3 a_{3}\right)+t_{0} h_{t}+\frac{h_{t}}{2} t_{2}-2 t_{2} h_{t} \mu=0, \\
& 24\left(B^{(t)}-\frac{h_{c}}{2} A^{(t)}\right) b_{3}+24\left(D^{(t)}-h_{c} B^{(t)}+\frac{h_{c}^{2}}{4} A^{(t)}\right) c 1_{3}+24\left(\frac{h_{c}}{2} B^{(t)}-\frac{h_{c}^{2}}{4} A^{(t)}\right) c 2_{3} \\
& -\bar{A}^{(t)}\left(c 1_{1}+4 a_{2}\right)+t_{1} h_{t}+\frac{3}{4} t_{3} h_{t}-6 t_{3} h_{t} \mu=0,4\left(\frac{h_{c}}{2} A^{(t)}+B^{(c)}-\frac{h_{c}}{2} A^{(b)}\right) b_{2} \\
& +2 h_{c}\left(B^{(t)}-\frac{h_{c}}{2} A^{(t)}\right) c 1_{2}+4\left(\frac{h_{c}^{2}}{4} A^{(t)}+D^{(c)}+\frac{h_{c}^{2}}{4} A^{(b)}\right) c 2_{2} \\
& -2 h_{c}\left(B^{(b)}+\frac{h_{c}}{2} A^{(b)}\right) c 3_{2}-\bar{A}^{(c)}\left(c 2_{0}+a_{1}+3 a_{3}\right)+\frac{h_{c}}{2} t_{0}+\frac{h_{c}}{4} t_{2}-t_{2} h_{c} \mu=0, \\
& 24\left(\frac{1}{2} h_{2} A^{(1)}+B^{(2)}-\frac{1}{2} h_{2} A^{(3)}\right) b_{3}+12 h_{2}\left(B^{(1)}-\frac{1}{2} h_{2} A^{(1)}\right) c 1_{3} \\
& +24\left(\frac{1}{4} h_{2}^{2} A^{(1)}+D^{(2)}+\frac{1}{4} h_{2}^{2} A^{(3)}\right) c 2_{3}-12 h_{2}\left(B^{(3)}+\frac{1}{2} h_{2} A^{(3)}\right) c 3_{3} \\
& -\bar{A}^{(2)}\left(c 2_{1}+4 a_{2}\right)+\frac{h_{c}}{2} t_{1}+\frac{3}{8} t_{3} h_{c}-3 t_{3} h_{c} \mu=0,4\left(B^{(b)}+\frac{h_{c}}{2} A^{(b)}\right) b_{2} \\
& -2 h_{c}\left(B^{(b)}+\frac{h_{c}}{2} A^{(b)}\right) c 2_{2}+4\left(\frac{h_{c}^{2}}{4} A^{(b)}+D^{(b)}+h_{c} B^{(b)}\right) c 3_{2} \\
& -\bar{A}^{(b)}\left(c 3_{0}+a_{1}+3 a_{3}\right)=0,24\left(B^{(b)}+\frac{h_{c}}{2} A^{(b)}\right) b_{3}-12 h_{c}\left(B^{(b)}+\frac{h_{c}}{2} A^{(b)}\right) c 2_{3} \\
& +24\left(\frac{h_{c}^{2}}{4} A^{(b)}+D^{(b)}+h_{c} B^{(b)}\right) c 3_{3}-\bar{A}^{(b)}\left(c 3_{1}+4 a_{2}\right)=0, \bar{A}^{(t)}\left(c 1_{1}+3 c 1_{3}\right)+\bar{A}^{(c)}\left(c 2_{1}+3 c 2_{3}\right) \\
& +\bar{A}^{(b)}\left(c 3_{1}+3 c 3_{3}\right)+4\left(\bar{A}^{(t)}+\bar{A}^{(c)}+\bar{A}^{(b)}\right) a_{2}-q_{0}-\frac{q_{2}}{2}+2 q_{2} \mu=0,4 \bar{A}^{(t)} c 1_{2}+4 \bar{A}^{(c)} c 2_{2}+4 \bar{A}^{(b)} c 3_{2}+24\left(\bar{A}^{(t)}+\bar{A}^{(c)}+\bar{A}^{(b)}\right) a_{3} \\
& -q_{1}-\frac{3}{4} q_{3}+6 q_{3} \mu=0
\end{aligned}
$$

The substitution of the expansions of Eqs. (40), (41) and (48) into the system of differential equations (22) yields an algebraic equation system. The first step will be to take the
In the similar way, using the recurrence relationships for the first and second derivative expansion coefficients from Eq. (11) and the Chebyshev expansions of Eqs. (40) and (41), 
the boundary conditions (37), (38) or (39) lead to the ten linear algebraic equations. For instance, (38) can be written as

Boundary conditions $C-F$ :
$+380 \times 10^{9}, g_{c}=1$ and $E_{b}=380 \times 10^{9}$. Then, the solutions to (50) plus the ten boundary equations $C-C, C-F$ or $C-S$ are, respectively, as

$$
\left\{\begin{array}{l}
b_{0}-b_{1}+b_{2}-b_{3}=0 \\
A^{(t)}\left(b_{1}+9 b_{3}+4 b_{2}\right) \\
+\left(B^{(t)}-\frac{h_{c}}{2} A^{(t)}\right)\left(c 1_{1}+9 c 1_{3}+4 c 1_{2}\right)+\frac{h_{c}}{2} A^{(t)}\left(c 2_{1}+9 c 2_{3}+4 c 2_{2}\right) \\
+A^{(c)}\left(b_{1}+9 b_{3}+4 b_{2}\right)+B^{(c)}\left(c 2_{1}+9 c 2_{3}+4 c 2_{2}\right)+A^{(b)}\left(b_{1}+9 b_{3}+4 b_{2}\right) \\
+\left(B^{(b)}+\frac{h_{c}}{2} A^{(b)}\right)\left(c 3_{1}+9 c 3_{3}+4 c 3_{2}\right)-\frac{h_{c}}{2} A^{(b)}\left(c 2_{1}+9 c 2_{3}+4 c 2_{2}\right)=0 \\
c 1_{0}-c 1_{1}+c 1_{2}-c 1_{3}=0 \\
B^{(t)}\left(b_{1}+9 b_{3}+4 b_{2}\right) \\
+\left(D^{(t)}-\frac{h_{c}}{2} B^{(t)}\right)\left(c 1_{1}+9 c 1_{3}+4 c 1_{2}\right)+\frac{h_{c}}{2} B^{(t)}\left(c 2_{1}+9 c 2_{3}+4 c 2_{2}\right) \\
-\frac{h_{c}}{2} A^{(t)}\left(b_{1}+9 b_{3}+4 b_{2}\right)-\frac{1}{2} h_{c}\left(B^{(1)}-\frac{1}{2} h_{c} A^{(1)}\right)\left(c 1_{1}+4 c 1_{2}+9 c 1_{3}\right) \\
-\frac{h_{c}^{2}}{4} A^{(t)}\left(c 2_{1}+4 c 2_{2}+9 c 2_{3}\right)=0, \\
c 2_{0}-c 2_{1}+c 2_{2}-c 2_{3}=0, \\
\frac{h_{c}}{2} A^{(t)}\left(b_{1}+9 b_{3}+4 b_{2}\right) \\
+\frac{h_{c}}{2}\left(B^{(t)}-\frac{h_{c}}{2} A^{(t)}\right)\left(c 1_{1}+9 c 1_{3}+4 c 1_{2}\right)+\frac{h_{c}^{2}}{4} A^{(t)}\left(c 2_{1}+9 c 2_{3}+4 c 2_{2}\right) \\
+B^{(c)}\left(b_{1}+9 b_{3}+4 b_{2}\right)+D^{(c)}\left(c 2_{1}+4 c 2_{2}+9 c 2_{3}\right)-\frac{h_{c}}{2} A^{(b)}\left(b_{1}+4 b_{2}+9 b_{3}\right) \\
-\frac{h_{c}}{2}\left(B^{(b)}+\frac{h_{c}}{2} A^{(b)}\right)\left(c 3_{1}+9 c 3_{3}+4 c 3_{2}\right)+\frac{h_{c}^{2}}{4} A^{(b)}\left(c 2_{1}+9 c 2_{3}+4 c 2_{2}\right)=0 \\
c 3_{0}-c 3_{1}+c 3_{2}-c 3_{3}=0, \\
B^{(b)}\left(b_{1}+9 b_{3}+4 b_{2}\right) \\
+\left(D^{(b)}+\frac{h_{c}}{2} B^{(b)}\right)\left(c 3_{1}+9 c 3_{3}+4 c 3_{2}\right)-\frac{h_{c}}{2} B^{(b)}\left(c 2_{1}+9 c 2_{3}+4 c 2_{2}\right) \\
+\frac{h_{c}}{2} A^{(b)}\left(b_{1}+9 b_{3}+4 b_{2}\right)+\frac{h_{c}}{2}\left(B^{(b)}+\frac{h_{c}}{2} A^{(b)}\right)\left(c 3_{1}+9 c 3_{3}+4 c 3_{2}\right) \\
-\frac{h_{c}^{2}}{4} A^{(b)}\left(c 2_{1}+9 c 2_{3}+4 c 2_{2}\right)=0 \\
a_{0}-a_{1}+a_{2}-a_{3}=0 \\
\bar{A}^{(t)}\left(c 1_{0}+c 1_{1}+c 1_{2}+c 1_{3}+a_{1}+9 a_{3}+4 a_{2}\right) \\
+\bar{A}^{(c)}\left(c 2_{0}+c 2_{1}+c 2_{2}+c 2_{3}+a_{1}+9 a_{3}+4 a_{2}\right) \\
+\bar{A}^{(b)}\left(c 3_{0}+c 3_{1}+c 3_{2}+c 3_{3}+a_{1}+9 a_{3}+4 a_{2}\right)=V . \\
\end{array}\right.
$$

Let $q=10^{7}, T=0, \mu=0, V=0, h_{t}=h_{b}=0.1, h_{c}=0.2$, $v_{t}=v_{c}=v_{b}=0.3, E_{t}=70 \times 10^{9}, E_{c}=-310 \times 10^{9}\left(\frac{z}{h_{c}}+\frac{1}{2}\right)^{g_{c}}$

$$
\left\{\begin{array}{l}
a=\left(-3.20021 \times 10^{-5}, 0,3.20021 \times 10^{-5}, 0\right), \\
b=\left(0,-4.83486 \times 10^{-6}, 0,4.83486 \times 10^{-6}\right), \\
c 1=\left(0,-7.23547 \times 10^{-5}, 0,7.23547 \times 10^{-5}\right), \\
c 2=\left(0,-6.57372 \times 10^{-5}, 0,6.57372 \times 10^{-5}\right), \\
c 3=\left(0,-9.93407 \times 10^{-5}, 0,9.93407 \times 10^{-5}\right),
\end{array}\right.
$$


Fig. 2 The values of $w_{25}$ for the $C-C$ sandwich beams regarded to the uniform load for various values of $N$

Fig. 3 The values of $w_{25}$ for sandwich beams regarded to the uniform load for different values of $K_{u}^{(s)}, K_{\varphi}^{(s)}$ and $K_{w}^{(s)}$
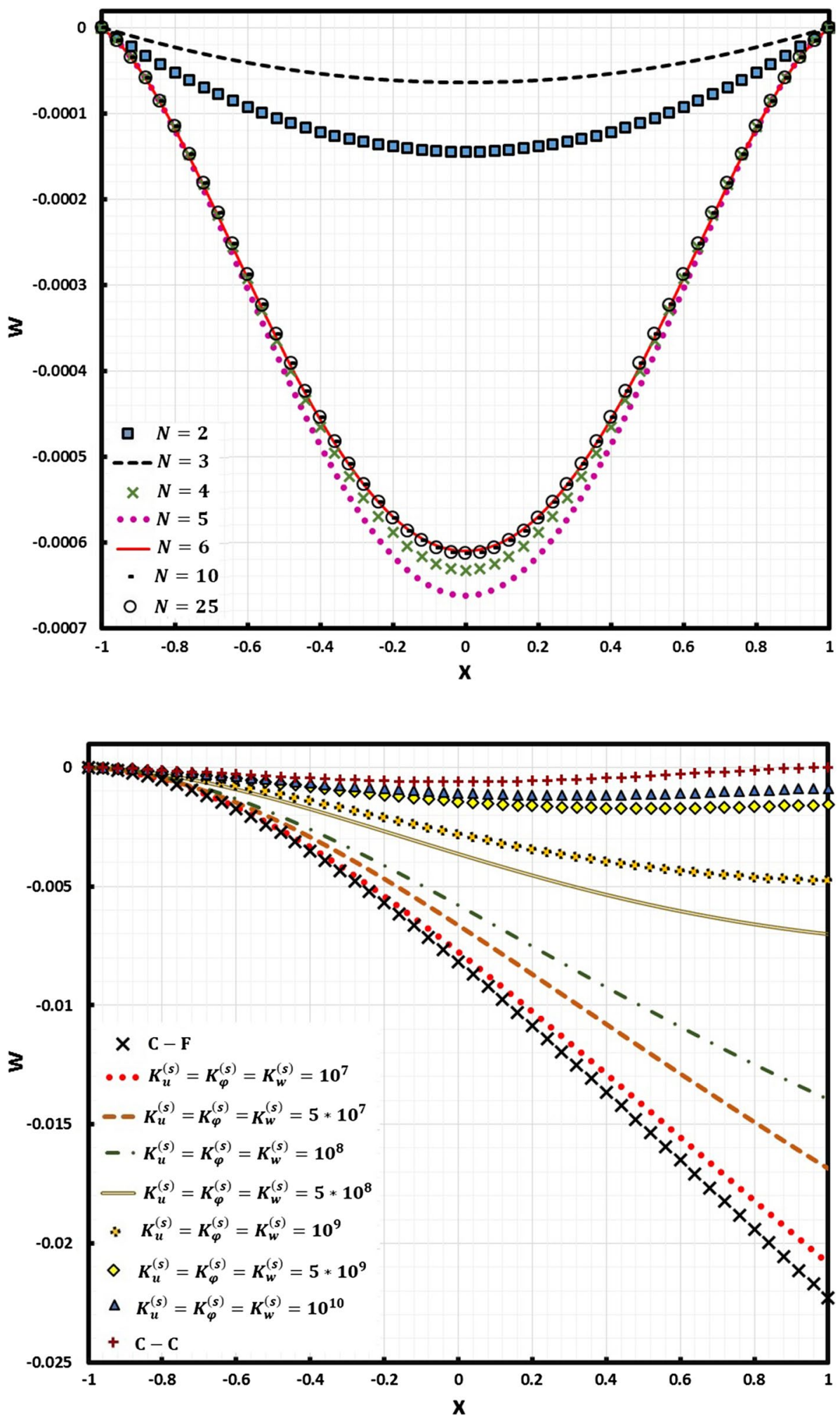
Fig. 4 The values of $w_{25}$ for sandwich beams regarded to the uniform load for $K_{w}^{(s)}=\infty\left(10^{100}\right)$ and different values of $K_{u}^{(s)}$ and $K_{\varphi}^{(s)}$

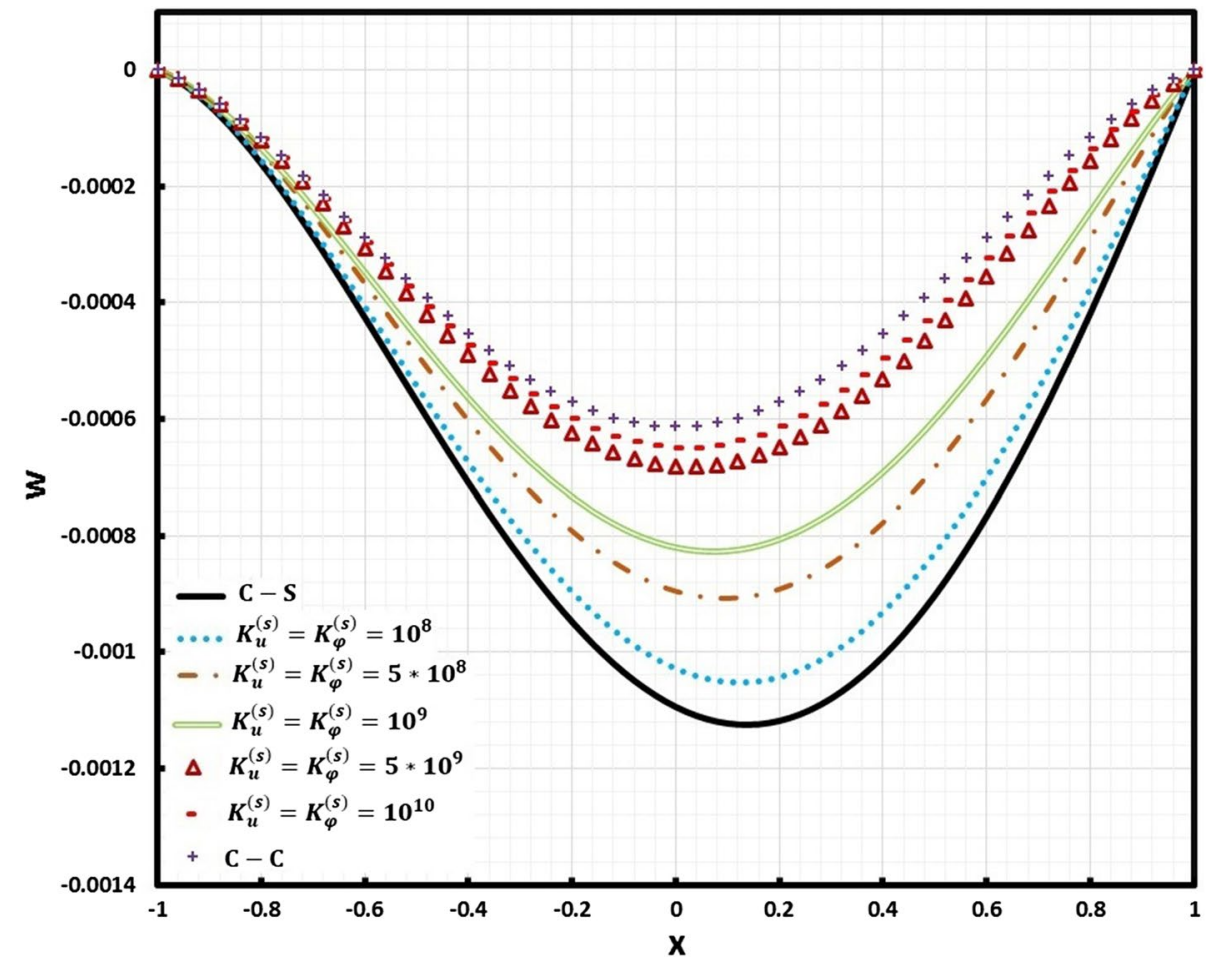

$\left\{\begin{array}{l}a=\left(-7.44699 \times 10^{-5},-1.95995 \times 10^{-5}, 7.44699 \times 10^{-5}, 1.95995 \times 10^{-5}\right), \\ b=\left(-3.29550 \times 10^{-6},-1.54451 \times 10^{-5},-7.22321 \times 10^{-6}, 4.92639 \times 10^{-6}\right), \\ c 1=\left(-5.35185 \times 10^{-5},-2.38879 \times 10^{-4},-1.09947 \times 10^{-4}, 7.54129 \times 10^{-5}\right), \\ c 2=\left(-5.50621 \times 10^{-5},-2.32667 \times 10^{-4},-1.05061 \times 10^{-4}, 7.25444 \times 10^{-5}\right), \\ c 3=\left(-4.61740 \times 10^{-5},-2.69457 \times 10^{-4},-1.33852 \times 10^{-4}, 8.94309 \times 10^{-5}\right) .\end{array}\right.$

Fig. 5 The values of $w_{25}$ for sandwich beams regarded to the uniform shear load for $K_{u}^{(s)}=K_{\varphi}^{(s)}=0$ and different values of $K_{w}^{(s)}$

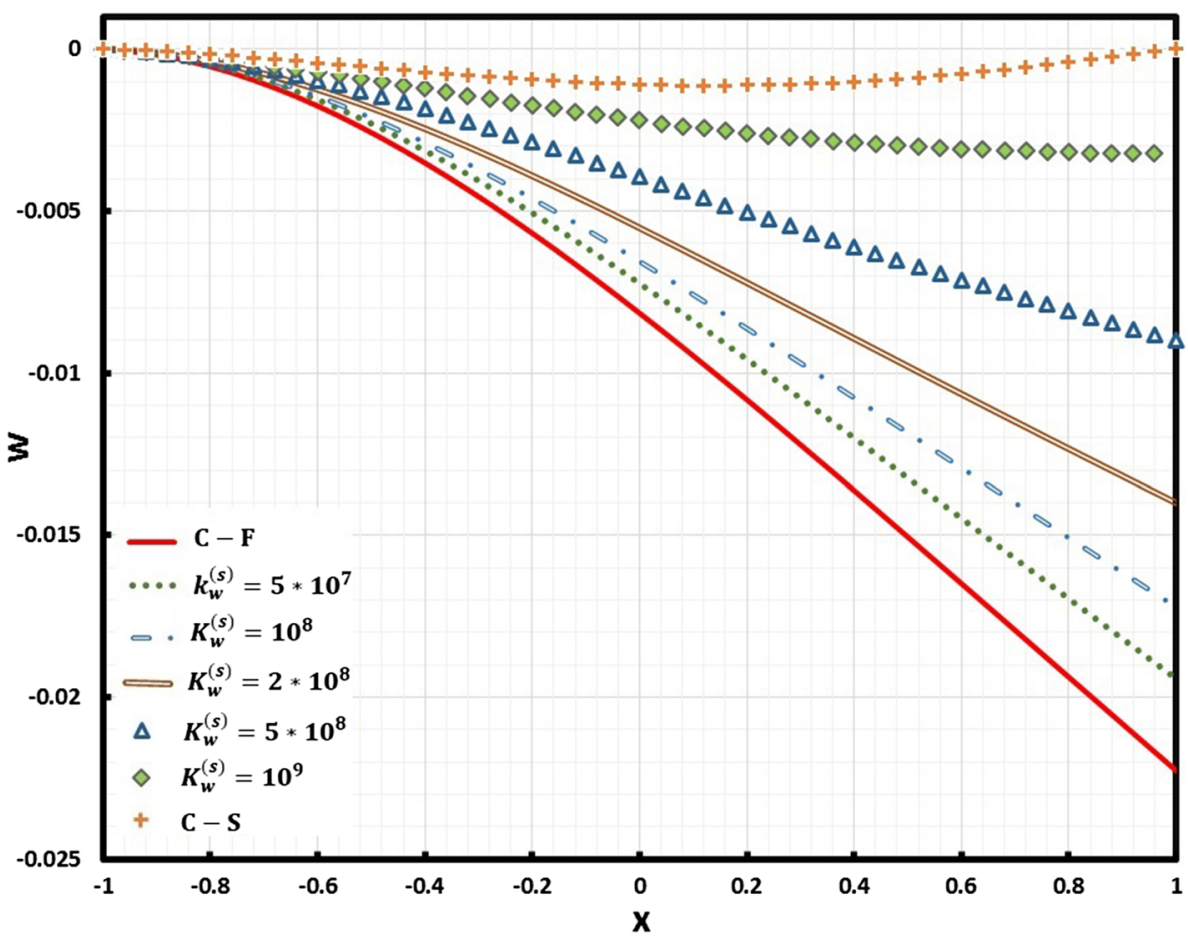


Table $1 w_{N}$ for clampedclamped $(C-C)$ end supports

\begin{tabular}{llllll}
\hline$x \mid N$ & 5 & 6 & 10 & 25 & 26 \\
\hline-1 & 0 & 0 & 0 & 0 & 0 \\
-0.8 & -0.000118317 & -0.000117006 & -0.000115237 & -0.000115240 & -0.000115240 \\
-0.6 & -0.000303853 & -0.000291497 & -0.000287530 & -0.000287644 & -0.000287644 \\
-0.4 & -0.000486476 & -0.000456357 & -0.000454298 & -0.000454177 & -0.000454177 \\
-0.2 & -0.000616088 & -0.000570078 & -0.000571434 & -0.000571412 & -0.000571412 \\
0 & -0.000662632 & -0.000610323 & -0.000613187 & -0.000613376 & -0.000613376 \\
0.2 & -0.000616088 & -0.000570078 & -0.000571434 & -0.000571412 & -0.000571412 \\
0.4 & -0.000486476 & -0.000456357 & -0.000454298 & -0.000454177 & -0.000454177 \\
0.6 & -0.000303853 & -0.000291497 & -0.000287530 & -0.000287644 & -0.000287644 \\
0.8 & -0.000118317 & -0.000117006 & -0.000115237 & -0.000115240 & -0.000115240 \\
1 & 0 & 0 & 0 & 0 & 0 \\
\hline
\end{tabular}

Table 2 Comparison the CTM and FEM results for $w_{25}$

\begin{tabular}{lllllll}
\hline & $C-C$ & $C-C$ & $C-F$ & $C-F$ & $C-S$ & $C-S$ \\
$x$ & CTM & FEM & CTM & FEM & CTM & FEM \\
\hline-1 & 0 & 0 & 0 & 0 & 0 & 0 \\
-0.8 & -0.000115240 & -0.000117742 & -0.00052494 & -0.00051581 & -0.000160906 & -0.00016286 \\
-0.6 & -0.000287644 & -0.000304835 & -0.00174432 & -0.00183505 & -0.000425826 & -0.000448896 \\
-0.4 & -0.000454177 & -0.000486541 & -0.00350617 & -0.00375483 & -0.000709959 & -0.000757281 \\
-0.2 & -0.000571412 & -0.000614643 & -0.00568032 & -0.00613143 & -0.000948686 & -0.00101680 \\
0 & -0.000613376 & -0.000660515 & -0.00815425 & -0.00884097 & -0.00109487 & -0.00117595 \\
0.2 & -0.000571412 & -0.000614643 & -0.0108328 & -0.0117789 & -0.00111867 & -0.00120239 \\
0.4 & -0.000454177 & -0.000486541 & -0.0136379 & -0.0148595 & -0.00100763 & -0.00108277 \\
0.6 & -0.000287644 & -0.000304835 & -0.0165092 & -0.0180165 & -0.000766492 & -0.000822802 \\
0.8 & -0.000115240 & -0.000117742 & -0.0194034 & -0.0212022 & -0.000417422 & -0.000447286 \\
1 & 0 & 0 & -0.0222944 & -0.0243880 & 0 & 0 \\
\hline
\end{tabular}

For $V=-0.4 \times 10^{7}$ and $q=0$, the solution to (50) plus the ten boundary equations $C-F(51)$ is:
The substitution of (52)-(55) into the (40) and (41), respectively, leads to

$$
\left\{\begin{array}{l}
\left(u_{0}\right)_{3}(x)=\left(-1.93394 \times 10^{-5}\right) x+\left(1.93394 \times 10^{-5}\right) x^{3} \\
\left(\varphi_{x}^{(t)}\right)_{3}(x)=\left(-2.89418 \times 10^{-4}\right) x+\left(2.89418 \times 10^{-4}\right) x^{3} \\
\left(\varphi_{x}^{(c)}\right)_{3}(x)=\left(-2.62948 \times 10^{-4}\right) x+\left(2.62948 \times 10^{-4}\right) x^{3} \\
\left(\varphi_{x}^{(b)}\right)_{3}(x)=\left(-3.97362 \times 10^{-4}\right) x+\left(3.97362 \times 10^{-4}\right) x^{3} \\
w_{3}(x)=\left(-6.40042 \times 10^{-5}\right)+\left(6.40042 \times 10^{-5}\right) x^{2},
\end{array}\right.
$$

$$
\left\{\begin{array}{l}
\left(u_{0}\right)_{3}(x)=\left(4.16078 \times 10^{-4}\right)+\left(2.45398 \times 10^{-4}\right) x \\
-\left(1.51487 \times 10^{-4}\right) x^{2}+\left(1.91919 \times 10^{-5}\right) x^{3}, \\
\left(\varphi_{x}^{(t)}\right)_{3}(x)=\left(6.55783 \times 10^{-3}\right)+\left(3.90877 \times 10^{-3}\right) x \\
-\left(2.37118 \times 10^{-3}\right) x^{2}+\left(2.77880 \times 10^{-4}\right) x^{3}, \\
\left(\varphi_{x}^{(c)}\right)_{3}(x)=\left(6.52837 \times 10^{-3}\right)+\left(3.93767 \times 10^{-3}\right) x \\
-\left(2.34196 \times 10^{-3}\right) x^{2}+\left(2.48748 \times 10^{-4}\right) x^{3}, \\
\left(\varphi_{x}^{(b)}\right)_{3}(x)=\left(6.71488 \times 10^{-3}\right)+\left(3.77801 \times 10^{-3}\right) x \\
-\left(2.51772 \times 10^{-3}\right) x^{2}+\left(4.19144 \times 10^{-4}\right) x^{3}, \\
w_{3}(x)=\left(-3.89890 \times 10^{-3}\right)-\left(6.73682 \times 10^{-3}\right) x \\
-\left(2.03178 \times 10^{-3}\right) x^{2}+\left(8.06148 \times 10^{-4}\right) x^{3},
\end{array}\right.
$$


Fig. 6 The values of $w_{25}$ for sandwich beams regarded to the uniform load for $K_{w}^{(s)}=10^{9}$ and different values of $K_{u}^{(s)}$ and $K_{\varphi}^{(s)}$

Fig. 7 The values of $w_{25}$ for sandwich beams regarded to the uniform load for different values of $K_{u}^{(s)}, K_{\varphi}^{(s)}$ and $K_{w}^{(s)}$
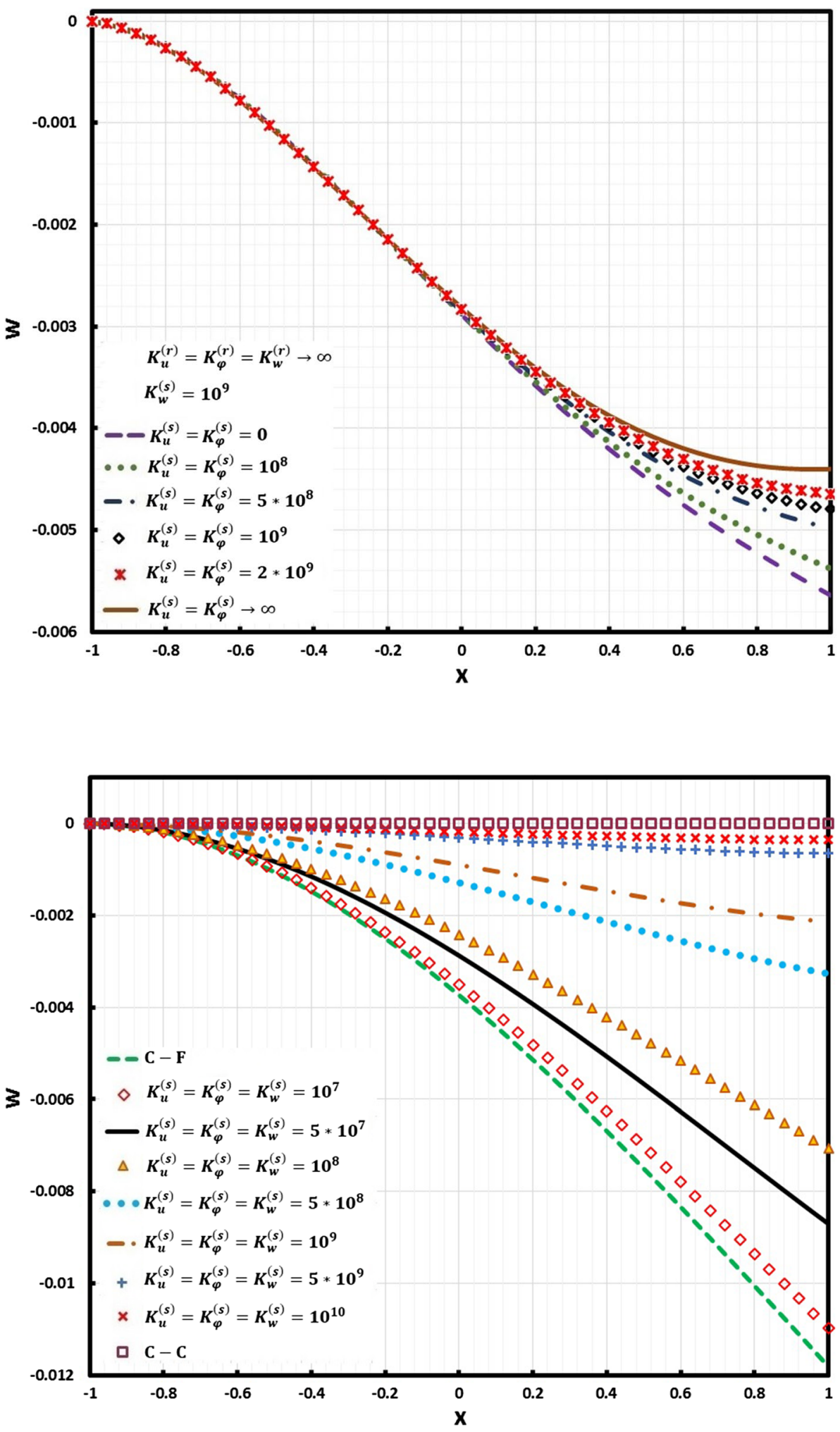


$$
\left\{\begin{array}{l}
\left(u_{0}\right)_{3}(x)=\left(3.92771 \times 10^{-6}\right)-\left(3.02243 \times 10^{-5}\right) x \\
-\left(1.44464 \times 10^{-5}\right) x^{2}+\left(1.97056 \times 10^{-5}\right) x^{3} \\
\left(\varphi_{x}^{(t)}\right)_{3}(x)=\left(5.64285 \times 10^{-5}\right)-\left(4.65118 \times 10^{-4}\right) x \\
-\left(2.19894 \times 10^{-4}\right) x^{2}+\left(3.01652 \times 10^{-4}\right) x^{3}, \\
\left(\varphi_{x}^{(c)}\right)_{3}(x)=\left(4.99989 \times 10^{-5}\right)-\left(4.50300 \times 10^{-4}\right) x \\
-\left(2.10122 \times 10^{-4}\right) x^{2}+\left(2.90178 \times 10^{-4}\right) x^{3}, \\
\left(\varphi_{x}^{(b)}\right)_{3}(x)=\left(8.76780 \times 10^{-5}\right)-\left(5.37750 \times 10^{-4}\right) x \\
\left(-2.67704 \times 10^{-4}\right) x^{2}+\left(3.57724 \times 10^{-4}\right) x^{3}, \\
w_{3}(x)=\left(-1.48940 \times 10^{-4}\right)-\left(7.83980 \times 10^{-5}\right) x \\
+\left(1.48940 \times 10^{-4}\right) x^{2}+\left(7.783980 \times 10^{-5}\right) x^{3} .
\end{array}\right.
$$

$$
\left\{\begin{array}{l}
\left(u_{0}\right)_{3}(x)=\left(4.06700 \times 10^{-4}\right)+\left(2.71978 \times 10^{-4}\right) x \\
-\left(1.35229 \times 10^{-4}\right) x^{2}-\left(5.06848 \times 10^{-7}\right) x^{3} \\
\left(\varphi_{x}^{(t)}\right)_{3}(x)=\left(6.41544 \times 10^{-3}\right)+\left(4.31605 \times 10^{-3}\right) x \\
-\left(2.12284 \times 10^{-3}\right) x^{2}-\left(2.34574 \times 10^{-5}\right) x^{3} \\
\left(\varphi_{x}^{(c)}\right)_{3}(x)=\left(6.39271 \times 10^{-3}\right)+\left(4.32995 \times 10^{-3}\right) x \\
-\left(2.10364 \times 10^{-3}\right) x^{2}-\left(4.08824 \times 10^{-5}\right) x^{3} \\
\left(\varphi_{x}^{(b)}\right)_{3}(x)=\left(6.53957 \times 10^{-3}\right)+\left(4.25870 \times 10^{-3}\right) x \\
\left(-2.22026 \times 10^{-3}\right) x^{2}+\left(6.06072 \times 10^{-5}\right) x^{3} \\
w_{3}(x)=\left(-3.70038 \times 10^{-3}\right)-\left(6.57038 \times 10^{-3}\right) x \\
-\left(2.15188 \times 10^{-3}\right) x^{2}+\left(7.18128 \times 10^{-4}\right) x^{3}
\end{array}\right.
$$

\section{Results and comparisons}

In the current section, different examples of FG micro/nanosandwich beams are investigated. To demonstrate the accuracy and reliability of the CTM, the obtained results of FG sandwich beam in especial cases (with classical edge conditions and $\mu=0$ ) are compared with the extracted results of ABAQUS software by using the finite element method. It can be mentioned that the extracted results from the ABAQUS software are obtained based on the 3D elasticity theory and the obtained results in this study are presented based on the theory of layerwise, so the difference between the results is expected. In all examples, it is assumed that $h_{t}=h_{b}=0.1, h_{c}=0.2, v_{t}=v_{c}=v_{b}=0.3, E_{t}=70 \times 10^{9}$, $E_{c}=-310 \times 10^{9}\left(\frac{z}{h_{c}}+\frac{1}{2}\right)^{g_{c}}+380 \times 10^{9}, g_{c}=1, E_{b}=380$ $\times 10^{9}$.

Example 1 In this example, results of FG sandwich beams subjected to $\mu=0, V=0, q=10^{7}$ and $T=0$ are obtained. Table 1 and Fig. 2 show the values of $w_{N}$ for clampedclamped edge $(C-C)$ and different amounts of $N$. It is observed that increasing the number of polynomial items $N$ improves the accuracy of results and leads to convergent solutions at $N=25$. Hence, $N=25$ is utilized in our numerical calculations. In Fig. 3, the values of in-plane translational, rotation and transverse translational stiffness parameters are supposed to be equal $\left(K_{u}^{(s)}=K_{\varphi}^{(s)}=K_{w}^{(s)}\right)$. Nine different
Fig. 8 Variations of the in-plane displacement regarded to the non-uniformly load for different values of $K_{u}^{(s)}, K_{\varphi}^{(s)}$ and $K_{w}^{(s)}$, $x=0.2$

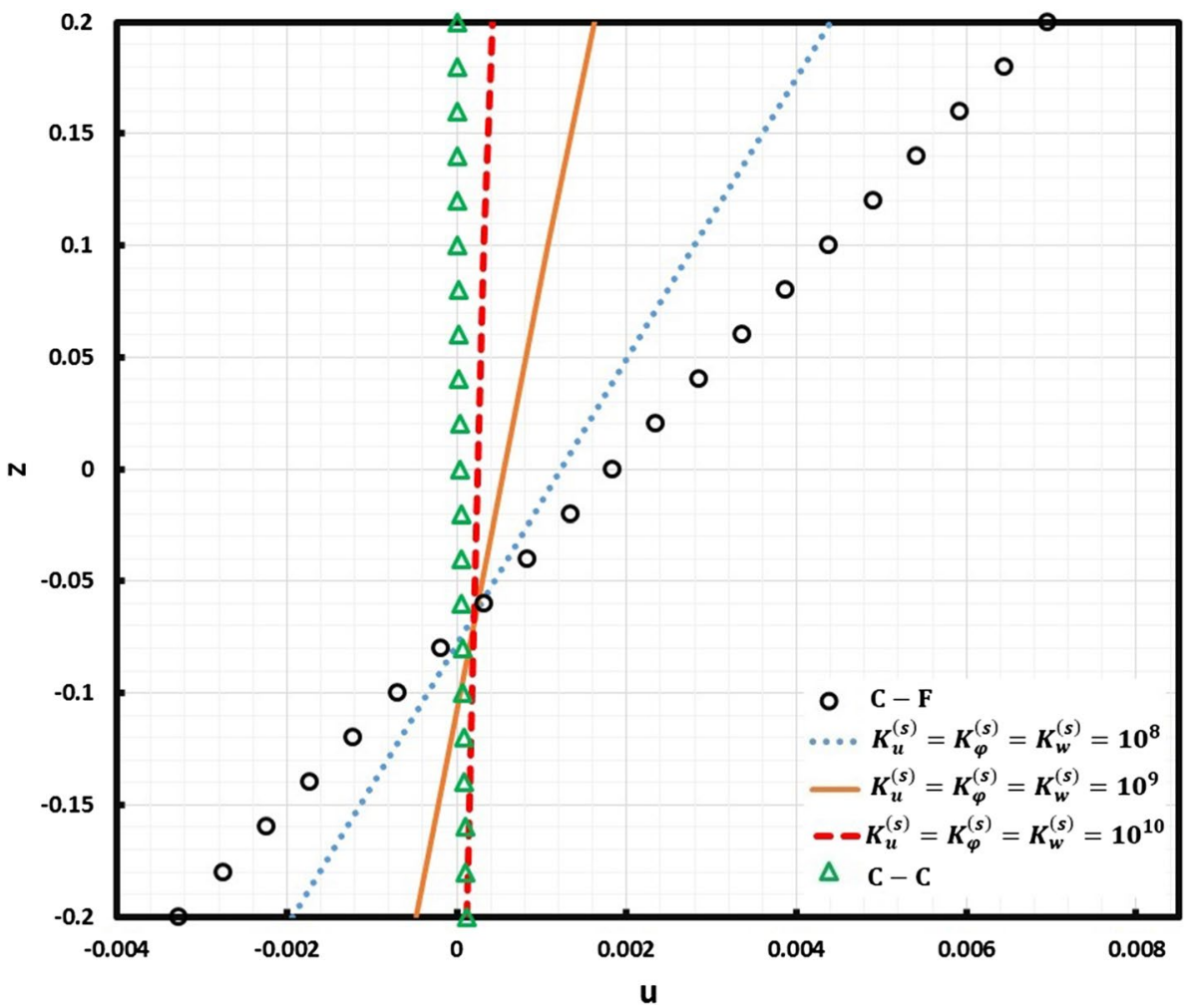


Fig. 9 Variations of the in-plane displacement regarded to the non-uniformly load for different values of $K_{u}^{(s)}, K_{\varphi}^{(s)}$ and $K_{w}^{(s)}$, $x=0.9$

Fig. 10 Variations of the inplane displacement regarded to the non-uniformly load for $K_{w}^{(s)}=\infty\left(10^{100}\right)$ and different values of $K_{u}^{(s)}$ and $K_{\varphi}^{(s)}, x=0.2$
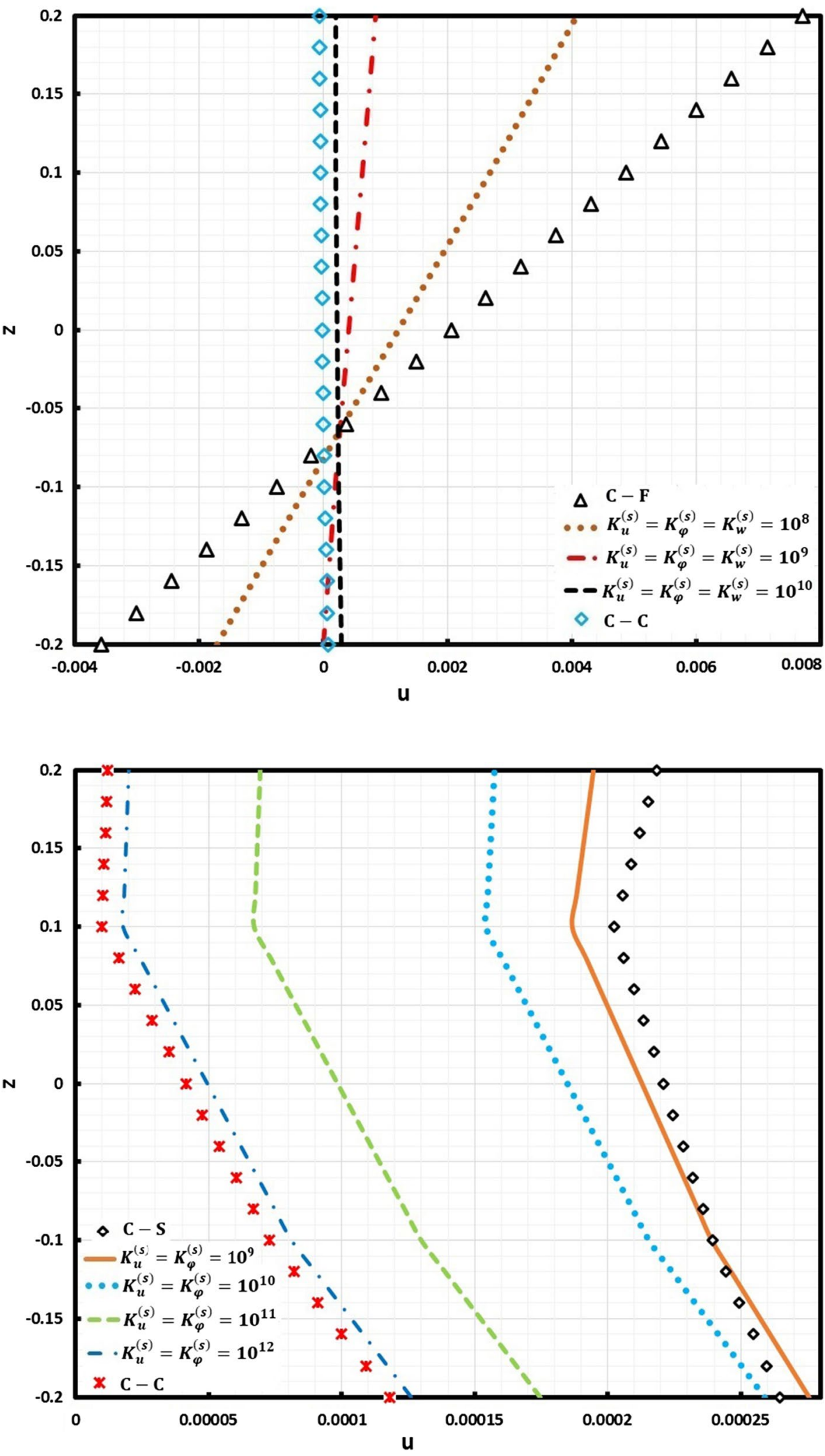

values, $K_{u}^{(s)}=K_{\varphi}^{(s)}=K_{w}^{(s)}=0, K_{u}^{(s)}=K_{\varphi}^{(s)}=K_{w}^{(s)}=\infty\left(10^{100}\right)$, $K_{u}^{(s)}=K_{\varphi}^{(s)}=K_{w}^{(s)}=10^{7}, 5 \times 10^{7}, 10^{8}, 5 \times 10^{8}$,
$K_{u}^{(s)}=K_{\varphi}^{(s)}=K_{w}^{(s)}=10^{7}, 5 \times 10^{7}, 10^{8}$, , are considered and variations of $w$ when the boundary condition tends from the 
Table 3 Comparison the CTM and FEM results regarded to $C-F$ edge for $w_{25}$

\begin{tabular}{lll}
\hline$x$ & CTM & FEM \\
\hline-1 & 0 & 0 \\
-0.8 & -0.00019272 & -0.00018583 \\
-0.6 & -0.00069808 & -0.00072984 \\
-0.4 & -0.00148046 & -0.00157826 \\
-0.2 & -0.00250520 & -0.00269294 \\
0 & -0.00373766 & -0.00403576 \\
0.2 & -0.00514322 & -0.00556869 \\
0.4 & -0.00668726 & -0.00725371 \\
0.6 & -0.00833516 & -0.00905274 \\
0.8 & -0.0100523 & -0.0109277 \\
1 & -0.0118040 & -0.0128451 \\
\hline
\end{tabular}

$C-F$ to the $C-C$ edge are demonstrated. It can be observed from Fig. 3 that increasing $K_{u}^{(s)}, K_{\varphi}^{(s)}$ and $K_{w}^{(s)}$ from zero to infinity decreases the lateral deflections. In Fig. 4, the values of in-plane translational and rotation stiffness parameters are supposed to be equal $\left(K_{u}^{(s)}=K_{\varphi}^{(s)}\right)$ and transverse translational stiffness parameter tends infinity $K_{w}^{(s)}=\infty\left(10^{100}\right)$. Seven different values $K_{u}^{(s)}=K_{\varphi}^{(s)}=0, K_{u}^{(s)}=K_{\varphi}^{(s)}=\infty\left(10^{100}\right)$, $K_{u}^{(s)}=K_{\varphi}^{(s)}=10^{8}, 5 \times 10^{8}, 10^{9}, 5 \times 10^{9}, 10^{10}$ are considered and variations of $w$ when the boundary condition tends from the $C-S$ to the $C-C$ edge are shown. It is seen from Fig. 4 that increasing $K_{u}^{(s)}$ and $K_{\varphi}^{(s)}$ from zero to infinity reduces the lateral deflections. In Fig. 5, the values of in-plane translational and rotation stiffness parameters are assumed to be $\left(K_{u}^{(s)}=K_{\varphi}^{(s)}=0\right)$. Seven different values $K_{w}^{(s)}=0$, $K_{w}^{(s)}=\infty\left(10^{100}\right), \quad K_{w}^{(s)}=5 \times 10^{7}, 10^{8}, 2 \times 10^{8}, 5 \times 10^{8}, 10^{9}$ are considered and variations of $w$ when the boundary condition tends from the $C-F$ to the $C-S$ edge are depicted. As it can be observed from Fig. 5, increasing $K_{w}^{(s)}$ from zero to infinity decreases the lateral deflections. In Fig. 6, the values of in-plane and rotation stiffness parameters are supposed to be equal $\left(K_{u}^{(s)}=K_{\varphi}^{(s)}\right)$ and transverse translational stiffness parameter is $K_{w}^{(s)}=10^{9}$. Six different values $K_{u}^{(s)}=K_{\varphi}^{(s)}=0$, $K_{u}^{(s)}=K_{\varphi}^{(s)}=\infty\left(10^{100}\right)$ $K_{u}^{(s)}=K_{\varphi}^{(s)}=10^{8}, 5 \times 10^{8}, 10^{9}, 2 \times 10^{9}$ are considered, and variations of $w$ are depicted. It may be observed from Fig. 6 that when $K_{u}^{(s)}$ and $K_{\varphi}^{(s)}$ increase from zero to infinity, the lateral deflections decrease. In Table 2, the lateral deflections at the $C-C, C-F$ and $C-S$ edges of the mentioned sandwich beams based on the CTM are compared with those extracted from the ABAQUS software based on the 3D theory of elasticity. Although the difference between the theories of Chebyshev tau method and ABAQUS software may causes some differences between the results of CTM and FEM, it can be observed from Table 2, there are good agreement between present results and results of the FEM for various edge conditions $C-C, C-F$ and $C-S$.
Fig. 11 Variations of the inplane displacement regarded to the non-uniformly load for $K_{w}^{(s)}=\infty\left(10^{100}\right)$ and different values of $K_{u}^{(s)}$ and $K_{\varphi}^{(s)}, x=0.5$

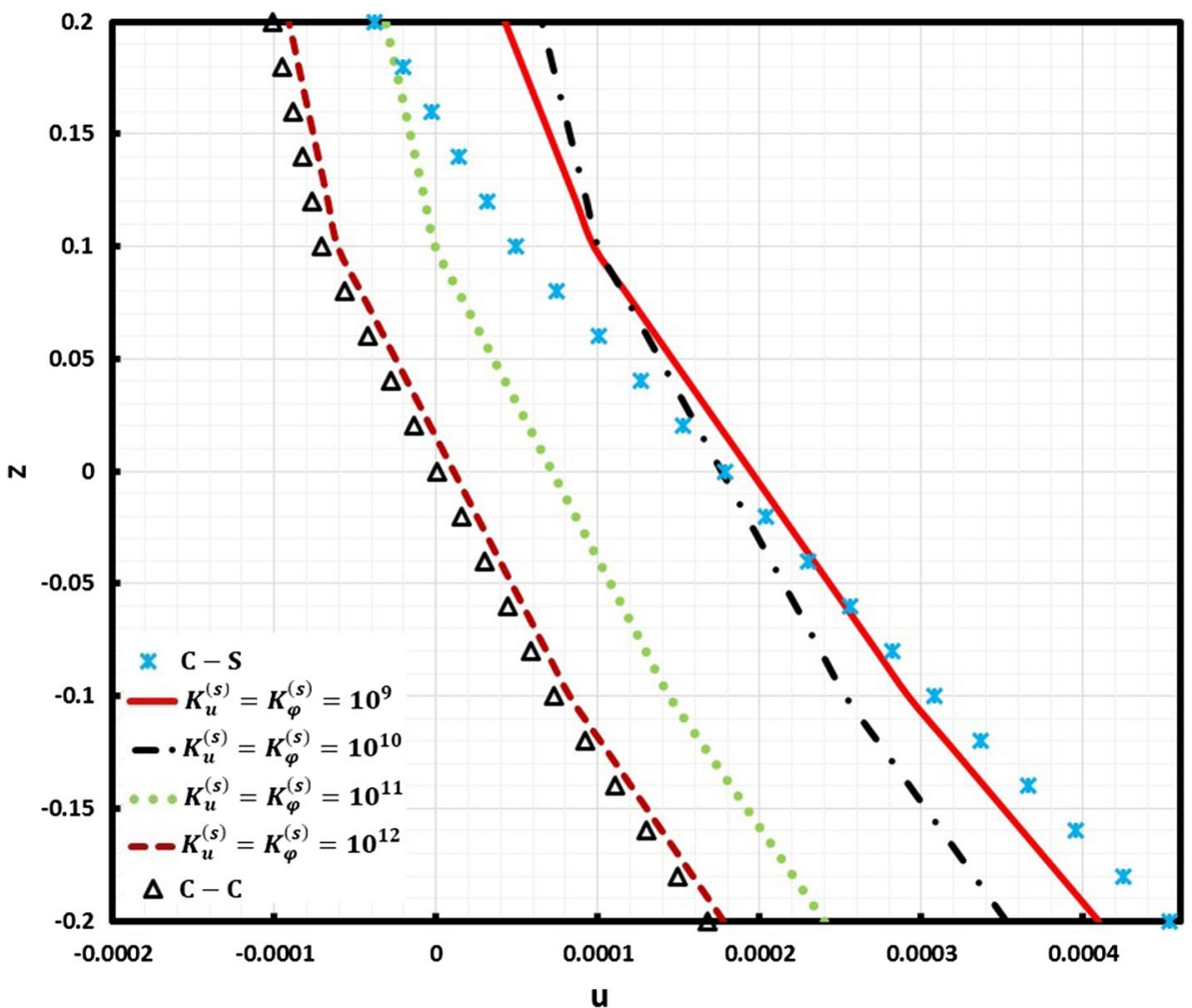


Fig. 12 Variations of the inplane displacement regarded to the non-uniformly load for $K_{w}^{(s)}=\infty\left(10^{100}\right)$ and different values of $K_{u}^{(s)}$ and $K_{\varphi}^{(s)}, x=0.9$

Fig. 13 Variations of the inplane displacement regarded to the non-uniformly load for $K_{u}^{(s)}=K_{\varphi}^{(s)}=0$ and different values of $K_{w}^{(s)}, x=0.2$
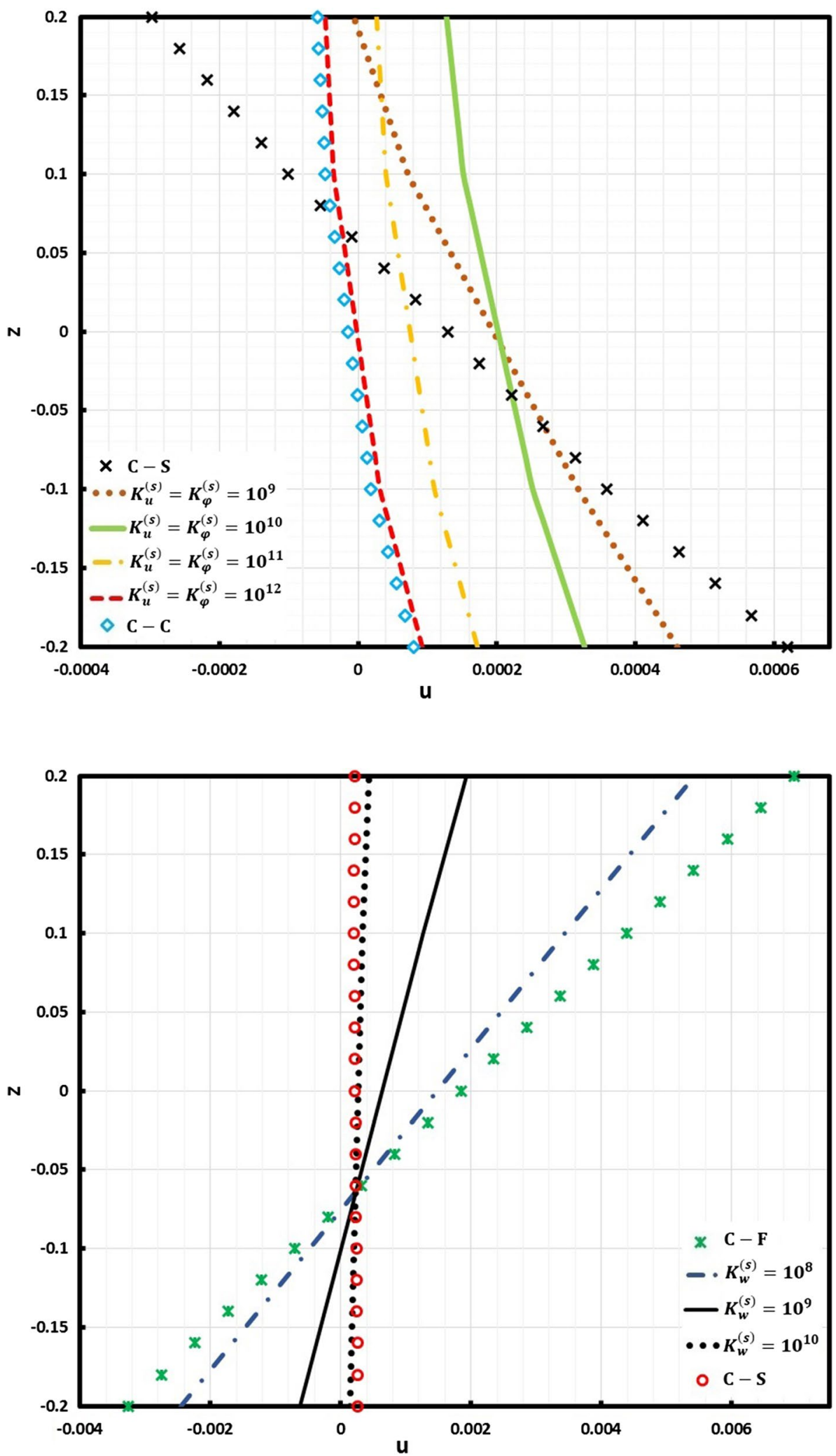
Fig. 14 Variations of the inplane displacement regarded to the non-uniformly load for $K_{u}^{(s)}=K_{\varphi}^{(s)}=0$ and different values of $K_{w}^{(s)}, x=0.9$
Fig. 15 The values of $w_{25}$ for $C-C$ sandwich beams regarded to the non-uniformly load for different values of $\mu$
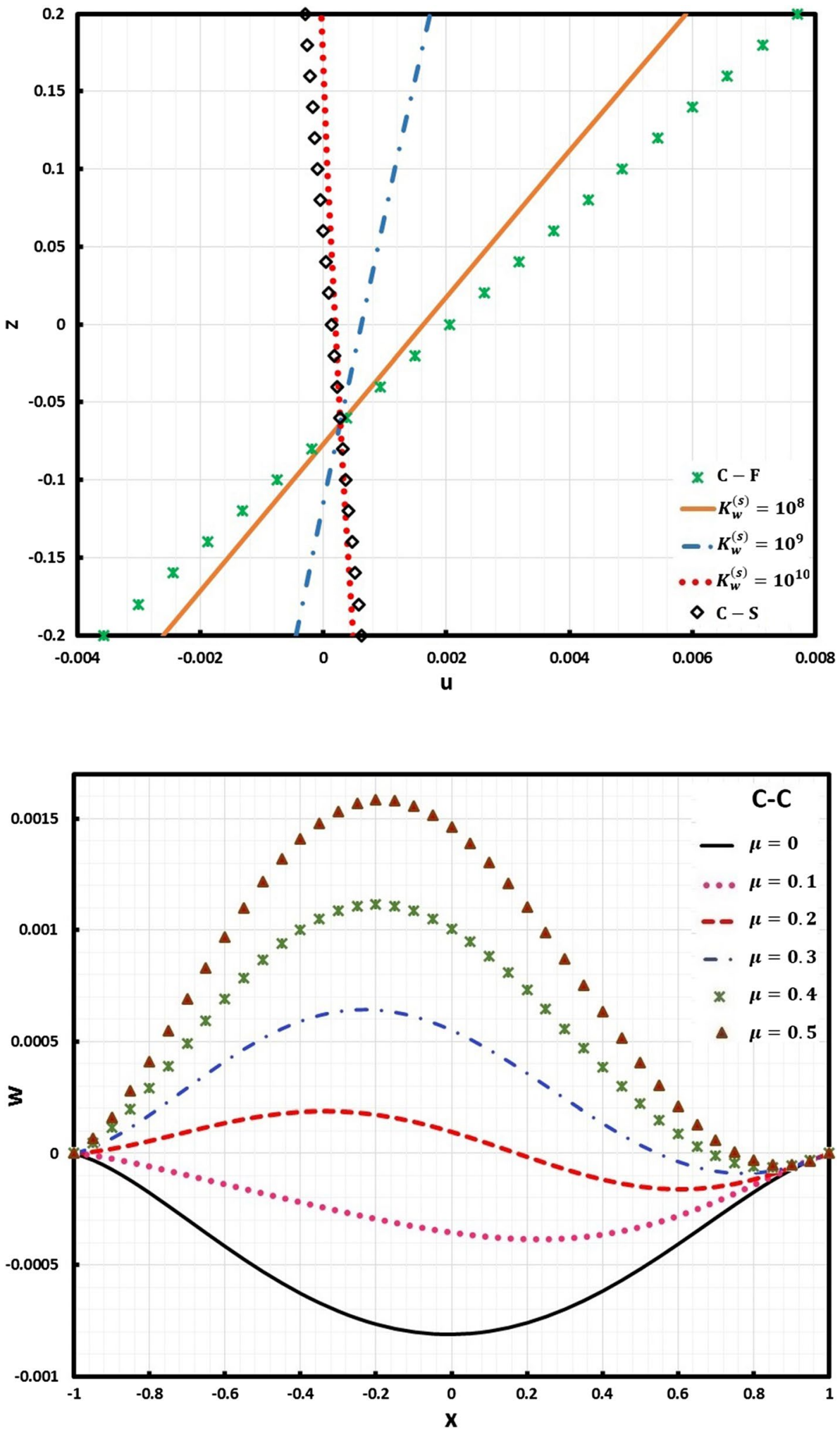

Example 2 In this example, results of FG sandwich beams subjected to $\mu=0, V=-0.4 \times 10^{7}, q=0$ and $T=0$ are obtained. In Fig. 7, the values of in-plane translational, rotation and transverse translational stiffness parameters are supposed to be equal $\left(K_{u}^{(s)}=K_{\varphi}^{(s)}=K_{w}^{(s)}\right)$. Nine different values $K_{u}^{(s)}=K_{\varphi}^{(s)}=K_{w}^{(s)}=0, K_{u}^{(s)}=K_{\varphi}^{(s)}=K_{w}^{(s)}=\infty\left(10^{100}\right)$, 
Fig. 16 The values of $w_{25}$ for $C-F$ sandwich beams regarded to the non-uniformly load for different values of $\mu$
Fig. 17 The values of $w_{25}$ for $C-S$ sandwich beams regarded to the non-uniformly load for different values of $\mu$
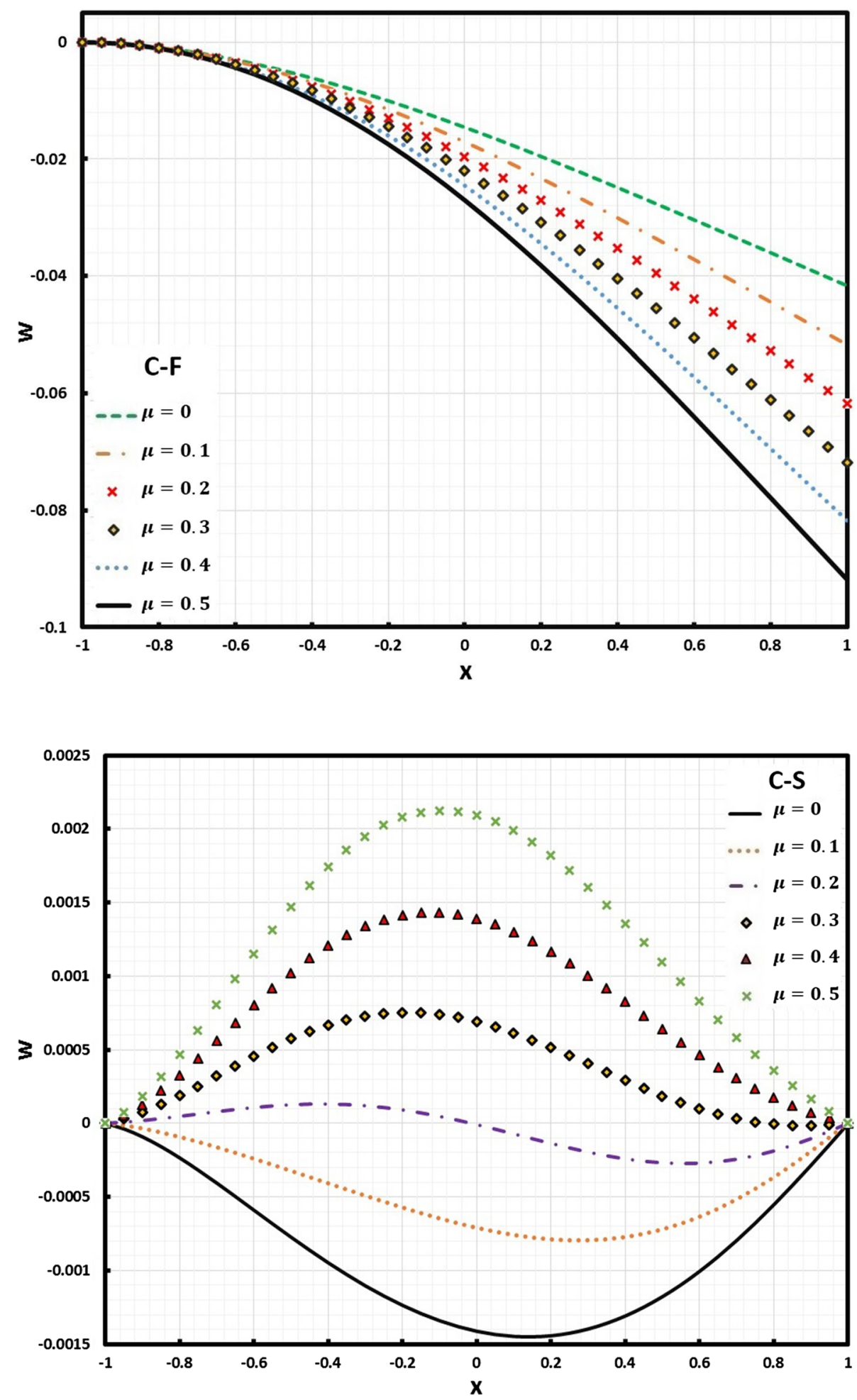

$K_{u}^{(s)}=K_{\varphi}^{(s)}=K_{w}^{(s)}=10^{7}, 5 \times 10^{7}, 10^{8}, 5 \times 10^{8}, 10^{9}, 5 \times 10^{9}, 10^{10}$ are considered and variations of $w$ when the boundary condition tends from the $C-F$ to the $C-F$ edge are shown. It is seen from Fig. 7 that increasing $K_{u}^{(s)}, K_{\varphi}^{(s)}$ and $K_{w}^{(s)}$ from zero to infinity reduces the lateral deflections. In Table 3, the lateral deflections at the $C-F$ edge of the mentioned sandwich beams based on the CTM are compared with those extracted from the ABAQUS software based on the 3D-elasticity. It is seen from Table 3, there are good agreement between present results and results of the FEM for $C-F$ boundary condition. 
Example 3 For analysis of sandwich beams regarded to $\mu=0, V=0$ and the non-uniformly normal load $q=T=\left(1+x+2 x^{2}-3 x^{3}\right) 10^{7}$ :

1. The values of in-plane, rotation and transverse translational stiffness parameters are assumed to be equal $\left(K_{u}^{(s)}=K_{\varphi}^{(s)}=K_{w}^{(s)}\right)$. Five different values $K_{u}^{(s)}=K_{\varphi}^{(s)}=K_{w}^{(s)}=0, K_{u}^{(s)}=K_{\varphi}^{(s)}=K_{w}^{(s)}=\infty\left(10^{100}\right)$, $K_{u}^{(s)}=K_{\varphi}^{\varphi(s)}=K_{w}^{(s)}=10^{8}, 10^{9}, 10^{10}$ are considered, and variations of the in-plane displacement when the boundary condition tends from the $C-F$ to the $C-C$ edge are plotted at $x=0.2$ and $x=0.9$ in Figs. 8 and 9 , respectively. It may be seen from Figs. 8 and 9 , increasing $K_{u}^{(s)}$, $K_{\varphi}^{(s)}$ and $K_{w}^{(s)}$ from zero to infinity reduces the in-plane displacements.

2. The values of in-plane and rotation stiffness parameters are supposed to be equal $\left(K_{u}^{(s)}=K_{\varphi}^{(s)}\right)$, and transverse translational stiffness parameter tends infinity $K_{w}^{(s)}=\infty\left(10^{100}\right)$. Six different values $K_{u}^{(s)}=K_{\varphi}^{(s)}=0$, $K_{u}^{(s)}=K_{\varphi}^{(s)}=\infty\left(10^{100}\right), \quad K_{u}^{(s)}=K_{\varphi}^{(s)}=10^{9}, 10^{10}, 10^{11}, 10^{12}$ are considered, and variations of the in-plane displacement when the boundary condition tends from the $C-S$ to the $C-C$ edge are shown at $x=0.2, x=0.5$ and $x=0.9$ in Figs. 10, 11 and 12 , respectively.

3. The values of in-plane translational and rotation stiffness parameters are supposed to be $\left(K_{u}^{(s)}=K_{\varphi}^{(s)}=0\right)$. Five different values $K_{w}^{(s)}=0, \quad K_{w}^{(s)}=\infty\left(10^{100}\right)$, $K_{w}^{(s)}=10^{8}, 10^{9}, 10^{10}$ are considered, and variations of the in-plane displacement when the boundary condition tends from the $C-F$ to the $C-S$ edge are depicted at $x=0.2$ and $x=0.9$ in Figs. 13 and 14 , respectively. It is seen from Figs. 13 and 14 , increasing $K_{w}^{(s)}$ from zero to infinity reduces the in-plane displacements.

Example 4 For analysis of FG micro/nano-sandwich beams subjected to $V=0$, the non-uniformly normal load $q=\left(1+x+2 x^{2}-3 x^{3}\right) 10^{7}$ and $T=\left(1+x+2 x^{2}-3 x^{3}\right) 10^{8}$ and six different values, $\mu=0,0.1,0.2,0.3,0.4,0.5$ are considered and variations of $w$ when the boundary conditions are the $C-C, C-F$ and $C-S$ edges, are shown in Figs. 15, 16 and 17 , respectively. When $\mu$ increases from zero to 0.5 , the lateral deflections of $C-C$ and $C-S$ sandwich beams decrease first and then increase in the positive direction of the coordinate axis, as it is seen from Figs. 15 and 17 . Also the lateral deflections of $C-F$ sandwich beams increase as shown in Fig. 16.

\section{Conclusion}

In this paper, Chebyshev tau method is employed for solution of governing equations of functionally graded micro/ nano-sandwich beams regarded to non-uniform normal and shear loads. The method leads to solving a system of linear algebraic equations. The obtained results reveal the accuracy, validity and applicability of the technique, even for sandwich beams under relatively complicated normal and shear loads. The comparisons reveal that there are good agreement between present results and results of the FEM for various edge conditions $C-C, C-F$ and $C-S$. In this work, MAPLE software is used to calculate the solutions obtained from the CT method.

Open Access This article is distributed under the terms of the Creative Commons Attribution 4.0 International License (http://creativecommons.org/licenses/by/4.0/), which permits unrestricted use, distribution, and reproduction in any medium, provided you give appropriate credit to the original author(s) and the source, provide a link to the Creative Commons license, and indicate if changes were made.

\section{References}

1. Canuto, C., Hussaini, M.Y., Quarteroni, A., Zang, T.A.: Spectral Methods: Fundamentals in Single Domains. Springer, Berlin (2006)

2. Kong, W., Wu, X.: Chebyshev tau matrix method for Poissontype equations in irregular domain. J. Comput. Appl. Math. 228, 158-167 (2009)

3. Lanczos, C.: Applied Analysis, pp. 464-517. Prentice-Hall, Englewood Cliffs (1964)

4. Johnson, D.: Chebyshev Polynomials in the Spectral Tau Method and Applications to Eigenvalue Problems. University of Florida Gainesville, Florida (1996)

5. Siyyam, H.I., Syam, M.I.: An accurate solution of the Poisson equation by the Chebyshev-tau method. J. Comput. Appl. Math. 85, 1-10 (1997)

6. Ahmadi, M.R., Adibi, H.: The Chebyshev Tau technique for the solution of Laplace's equation. Appl. Math. Comput. 84, 895-900 (2007)

7. Saadatmandi, A., Dehghan, M.: Numerical solution of hyperbolic telegraph equation using the Chebyshev tau method. Numer. Methods Partial Differ. Equ. 26, 239-252 (2010)

8. Wang, H.: An efficient Chebyshev-Tau spectral method for Ginzburg-Landau-Schrödinger equations. Comput. Phys. Commun. 181, 325-340 (2010)

9. Lee, J.: Application of Chebyshev-tau method to the free vibration analysis of stepped beams. Int. J. Mech. Sci. 101-102, 411-420 (2015)

10. Eltaher, M.A., Khairy, A., Sadoun, A.M., Omar, F.A.: Static and buckling analysis of functionally graded Timoshenko nanobeams. Appl. Math. Comput. 229, 283-295 (2014)

11. Foudal, N., El-midany, T., Sadoun, A.M.: Bending, buckling and vibration of a functionally graded porous beam using finite elements. J. Appl. Comput. Mech. 3, 274-282 (2017) 
12. Sobczak, J., Drenchev, L.: Functionally Graded Materials Processing and Modeling. Motor Transport Institute-Warsaw Foundry Research Institute, Cracow (2008)

13. Smith, B., Szyniszewski, S., Hajjar, J., Schafer, B., Arwade, S.: Steel foam for structures: A review of applications, manufacturing and material properties. J. Constr. Steel Res. 71, 1-10 (2012)

14. Ashby, M.F., Evans, T., Fleck, N.A., Hutchinson, J., Wadley, H., Gibson, L.: Metal Foams: A Design Guide. Elsevier, Amsterdam (2000)

15. Rabiei, A., Vendra, L.: A comparison of composite metal foam's properties and other comparable metal foams. Mater. Lett. 63, 533-536 (2009)

16. Lim, T.J., Smith, B., McDowell, D.: Behavior of a random hollow sphere metal foam. Acta Mater. 50, 2867-2879 (2002)

17. Raj, S., Ghosn, L., Lerch, B., Hebsur, M., Cosgriff, L., Fedor, J.: Mechanical properties of 17-4PH stainless steel foam panels. Mater. Sci. Eng., A 456, 305-316 (2007)

18. Park, C., Nutt, S.: Anisotropy and strain localization in steel foam. Mater. Sci. Eng., A 299, 68-74 (2001)

19. Badiche, X., Forest, S., Guibert, T., Bienvenu, Y., Bartout, J.D., Ienny, P., Croset, M., Bernet, H.: Mechanical properties and nonhomogeneous deformation of open-cell nickel foams: application of the mechanics of cellular solids and of porous materials. Mater. Sci. Eng., A 289, 276-288 (2000)

20. Gibson, L.: Mechanical behavior of metallic foams. Annu. Rev. Mater. Sci. 30, 191-227 (2000)

21. Kwon, Y., Cooke, R., Park, C.: Representative unit-cell models for open-cell metal foams with or without elastic filler. Mater. Sci. Eng., A 343, 63-70 (2003)

22. Sanders, W., Gibson, L.: Mechanics of hollow sphere foams. Mater. Sci. Eng., A 347, 70-85 (2003)

23. Warren, W., Kraynik, A.: The linear elastic properties of open-cell foams. J. Appl. Mech. 55, 341-346 (1988)

24. Banhart, J.: Manufacture, characterisation and application of cellular metals and metal foams. Progr. Mater. Sci. 46, 559-632 (2001)

25. Avalle, M., Belingardi, G., Montanini, R.: Characterization of polymeric structural foams under compressive impact loading by means of energy-absorption diagram. Int. J. Impact Eng. 25, 455-472 (2001)

26. Rajendran, R., Sai, K.P., Chandrasekar, B., Gokhale, A., Basu, S.: Preliminary investigation of aluminium foam as an energy absorber for nuclear transportation cask. Mater. Des. 29, 17321739 (2008)

27. Mortensen, A., Suresh, S.: Functionally graded metals and metalceramic composites. 1. Process. Int. Mater. Rev. 40, 239-265 (1995)

28. Chen, D., Yang, J., Kitipornch, S.: Elastic buckling and static bending of shear deformable functionally graded porous beam. Compos. Struct. 133, 54-61 (2015)

29. Mason, J.C., Handscomb, D.C.: Chebyshev Polynomials. Chapman and Hall/CRC, New York (2003)

30. Gardner, D.R., Trogdon, S.A.: A modified tau spectral method that eliminates spurious eigenvalues. J. Comput. Phys. 80, 137-167 (1989)

31. Lanczos, C.: Applied Analysis. Prentice Hall Inc., Englewood Cliffs (1956)

32. Fox, L.: Chebyshev methods for ordinary differential equations. Comput. J. 4, 318-331 (1962)

33. Gottlieb, D., Orszag, S.A.: Numerical Analysis of Spectral Methods. SIAM, Philadelphia (1977)

34. Orszag, S.A.: Galerkin approximations to flows within slabs, spheres, and cylinders. Phys. Rev. Lett. 26, 1100 (1971)

35. Orszag, S.A.: Accurate solution of the Orr-Sommerfeld stability equation. J. Fluid Mech. 50, 689-703 (1971)

36. Alipour, M.M.: Effects of elastically restrained edges on FG sandwich annular plates by using a novel solution procedure based on layerwise formulation. Arch. Civ. Mech. Eng. 16, 678-694 (2016)

37. Alipour, M.M., Shariyat, M.: Analytical layerwise stress and deformation analysis of laminated composite plates with arbitrary shapes of interfacial imperfections and discontinuous lateral deflections. Compos. Struct. 200, 88-102 (2018)

38. Alipour, M.M.: A novel economical analytical method for bending and stress analysis of functionally graded sandwich circular plates with general elastic edge conditions, subjected to various loads. Compos. B Eng. 95, 48-63 (2016)

Publisher's Note Springer Nature remains neutral with regard to jurisdictional claims in published maps and institutional affiliations. 